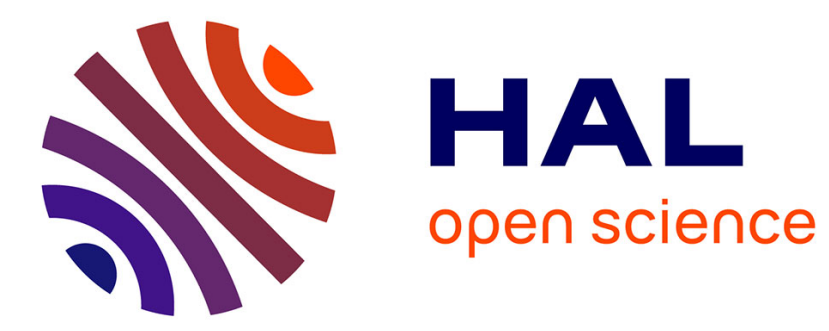

\title{
Information-Movement Coupling in The Control of Driver Approach to an Intersection
}

Julie Mathieu, Reinoud J. Bootsma, Catherine Berthelon, Gilles Montagne

\section{To cite this version:}

Julie Mathieu, Reinoud J. Bootsma, Catherine Berthelon, Gilles Montagne. Information-Movement Coupling in The Control of Driver Approach to an Intersection. Ecological Psychology, 2017, 4 (29), pp. 317-341. 10.1080/10407413.2017.1369853 . hal-01626260

\section{HAL Id: hal-01626260 https://hal.science/hal-01626260}

Submitted on 30 Oct 2017

HAL is a multi-disciplinary open access archive for the deposit and dissemination of scientific research documents, whether they are published or not. The documents may come from teaching and research institutions in France or abroad, or from public or private research centers.
L'archive ouverte pluridisciplinaire HAL, est destinée au dépôt et à la diffusion de documents scientifiques de niveau recherche, publiés ou non, émanant des établissements d'enseignement et de recherche français ou étrangers, des laboratoires publics ou privés. 


\section{Information-movement coupling in the control of driver}

\section{approach to an intersection}

\section{Introduction}

Recent data on driving-related road accidents (e.g., the 2015 assessment carried out by the French Observatoire National Interministériel de la Sécurité Routière [National Interministerial Road Safety Observatory]) showed that approach and crossing maneuvers at intersections were particularly prone to accidents; indeed in the year 2015 3,461 people were killed and 70,802 people were injured in France during such maneuvers, the gravity of these accidents being due to the violence of the collisions. Identifying the factors that can explain such accidents is a major societal issue, in order to develop and implement effective prevention policies. Our study was part of this process; specifically, because it aimed at gaining a better understanding of the perceptual-motor mechanisms that underlie an intersection crossing task.

The present contribution built on earlier work from our group on the active control of speed during approach to an intersection with incoming traffic (Louveton, Bootsma, Guerrin, Berthelon \& Montagne, 2012; Louveton, Montagne, Berthelon \& Bootsma, 2012; Matthieu, Bootsma, Berthelon \& Montagne, 2017). Driving a simulator, the participants' task in these studies was to safely cross the intersection by passing through a gap in the train of incoming traffic. In order to evoke adjustments in driving speed, on each trial the initial participantintersection distance was varied, so that without any speed adjustments they would arrive at the intersection ahead (Early Offset), behind (Late Offset) or at the same time (No Offset) as 
the center of the traffic gap. The results consistently demonstrated that, when required, approach to the intersection gave rise to gradual adjustments in driving speed with participants targeting a precise, limited crossing zone located slightly ahead of the center of the traffic gap. Overall, our previous results revealed that different task variables (traffic gap size and intersection geometry: Louveton, Bootsma et al., 2012; leading and trailing traffic gap vehicles’ speed: Louveton, Montagne et al., 2012; traffic gap vehicles’ size and type: Mathieu et al., 2017) subtly but systematically affected the evolution of driving speed during approach to the intersection. Yet, in all cases, crossing the intersection remained circumscribed to an area slightly ahead of the center of the traffic gap. Adjustments in approach speed thus appeared to indicate an attempt to optimize performance on the task, by targeting a 'low-risk' crossing zone, located around the center of the gap, and prioritizing the earlier part (see Chihak, Plumert, Ziemer, Babu, Grechkin, Cremer \& Kearney, 2010; Chihak, Grechkin, Kearnev, Cremer \& Plumert, 2014 for equivalent results from an intersection crossing task on a bicycle).

A complementary analysis, based on a prospective variable denoted current deviation, allowed us to more directly evaluate the functional character of observed speed adjustments. At any moment during the approach to the intersection, current deviation corresponds to the (spatial or temporal) distance from the center of the traffic gap at which the participant would pass the intersection if the current driving speed were to remain constant from thereon (Louveton, Bootsma et al., 2012). This future-related variable thus allowed determining whether speed adjustments served to reduce current deviation, that is, to continuously target the center of the gap in the train of incoming traffic. The results obtained indeed confirmed the functional character of speed adjustments: they resulted in a gradual reduction in current deviation during the approach phase, before ultimately targeting a zone slightly offset in the direction of the gap's leading vehicle during the last instances before intersection crossing. 
The repeated observation of this same pattern of results under varying task conditions (Louveton, Bootsma et al., 2012; Louveton, Montagne et al., 2012; Mathieu et al., 2017) points to the operation of a control mechanism that is based on a continuous informationmovement coupling (Bootsma, 1998) aimed at gradually modulating speed, in such a way that the adjustments necessary to carry out the task were distributed over the whole approach phase. Comparing active control of approach with discrete judgments of time remaining until arrival, in Mathieu et al. (2017) we made a further step in the characterization of this control mechanism by showing that, contrary to conventional thinking, control of speed during approach to the intersection was not based on successive estimates of the time remaining until arrival (necessarily inaccurate for long durations, see Schiff \& Detwiller, 1979; McLeod \& Ross, 1983), but on a precise perception of the state of the agent-environment system, as captured by the current deviation.

These results were thus consistent with the precepts of the ecological approach (Gibson, 1986; Bootsma, 1998; Warren, 1988, 2006) according to which the control of a goal-directed action is based on a coupling between an information variable that specifies the state of the agentenvironment system (AES) and a movement variable, influencing this AES state. Control of the action can then be conceived as a circular process in which behavior-related adjustments derive from the reciprocal relations between information and movement. By means of laws of control (Warren, 1988), mathematical formalizations of such reciprocal relationships provide testable designs of the information-movement relationship underlying the control of an action (see, for instance, Warren, Young \& Lee, 1986; Montagne, Laurent, Durey, \& Bootsma, 1999; Warren, Kay, Zosh, Duchon \& Sahuc, 2001; Chardenon, Montagne, Laurent \& Bootsma, 2004). 
However, the formalization of a control law is not always easy, a fortiori when task constraints are complex, as is the case for intersection crossing tasks in which the intervehicular traffic gap offers the agent considerable margins for maneuver. In this case, it becomes difficult to determine whether the limits of the inter-vehicular gap and/or the gap taken as a whole are taken into account by the agent (Louveton, Montagne et al., 2012). Consequently, while the formalization of a control law for this kind of task remains a longterm objective, in the present study we concentrated on extending the analysis of the regulatory behavior of drivers in our intersection-crossing task. This analysis allowed us to more precisely characterize regulation behavior, and thereby provide recommendations for studies that seek to formalize a control law.

Apart from our own work and that of Chihak and Plumert, few studies have attempted to characterize the perceptual-motor control mechanisms that underlie active intersectioncrossing tasks (cf. Mathieu et al., 2017). However, many sports skills, such as the standing backward somersault in gymnastics (Bardy \& Laurent, 1998), the hitting or catching of a ball (Bootsma \& van Wieringen, 1990), or the long jump run-up (Lee, Lishman \& Thomson, 1982), have been analyzed in terms of the reciprocal relations between information and movement, and various markers of the information-movement coupling have been identified.

The first marker corresponds to a gradual improvement in the precision of the action over the course of execution. This progressive gain in precision has been observed in the context of the long jump run-up, described in terms of a gradual decrease in the amount of adjustment required to precisely strike the takeoff board (Montagne, Cornus, Glize, Quaine \& Laurent, 2000). It has also been highlighted in ball-catching tasks, based on the convergence of the current speed of hand movement towards the speed required to successfully complete the task (Peper, Bootsma, Mestre \& Bakker, 1994; Montagne et al., 1999). Similarly, the above- 
discussed progressive reduction in current deviation over the approach to an intersection observed in our earlier work (Louveton, Bootsma et al., 2012; Louveton, Montagne et al., 2012; Mathieu et al., 2017) could be an indication of the operation of information-movement coupling.

The second, somewhat subtler, marker of information-movement coupling resides in the time course of variability over repeated trials (i.e., the evolution during task execution of withinparticipant between-trial variability) in both intermediate adjustment and task-level control variables. Supposing that task execution does not change qualitatively over a limited number of repeated trials, between-trial variability patterns allow inferences to be made about the control mechanisms operating at the scale of a trial, as detailed below.

In the long jump run-up, one task-level variable that determines performance on the task corresponds to the distance between the toes of the push-off leg's foot from the front edge of the takeoff board. At the last point of contact with the ground before push-off, this distance determines the final error with respect to the takeoff board and thereby, at least in part, subsequent long-jump performance. This toe-to-board distance can, of course, also be calculated for each previous step of the run-up. The data in the literature indicate that between-trial variability in the toe-to-board distance decreased over the last steps prior to takeoff and was minimal for the very last step that precedes takeoff (e.g., Lee et al., 1982; Scott, Li \& Davids, 2000), reflecting the visual adjustment of the length (or duration) of the step. Between-trial variability in step length (i.e., the intermediate adjustment variable) increased concomitantly with the decrease in the between-trial variability of the task-level control variable toe-to-board distance. In terms of control, these results indicated that tasklevel errors accumulated during the first part of the run-up were compensated for in the final 
steps, via the adjustment of step length, in order to hit the takeoff board as accurately as possible.

Similar results were obtained by Bardy and Laurent (1998) in a study of the standing backward somersault performed by expert gymnasts. Here, the (task-level) control variable determining task success corresponds to the orientation of the body in space, and the intermediate adjustment variables correspond to the thigh-leg and trunk-thigh angles that modulate the speed of rotation of the body in space and consequently its orientation. The results obtained once again showed opposite variations in the patterns of between-trial variability of the adjustment (intermediate) and control (task-level) variables during the execution of the somersault. The approach to the landing was characterized by increased between-trial variability in the trunk-thigh and thigh-leg angles, while between-trial variability in the orientation of the body in space decreased and became minimal at the time of landing. The speed of body rotation was therefore controlled via the modulation of the opening or closing of the above-mentioned angles in order to orient the body in space into a position that was optimal for landing stabilization.

In our previous studies (i.e., Louveton, Bootsma et al., 2012; Louveton, Montagne, et al., 2012; Mathieu et al., 2017), the regulation behavior of participants was analyzed through the inspection of average kinematic patterns; our analyses did not focus on the time course of variability over repeated trials in both intermediate and task level variables. If mechanisms, equivalent to those described above, were used in the intersection-crossing task, between-trial variability in (intermediate adjustment variable) speed may be expected to increase, while between-trial variability in (task-level control variable) current deviation may be expected to concomitantly decrease over the approach to the intersection. 
Bootsma, Houbiers, Whiting and Van Wieringen (1991) used the metaphor of a funnel-like control to illustrate the change in "tolerance" for between-trial variability in movement patterns during execution (Figure 1). According to the data presented above, this tolerance is high under mild time constraints, reduces with time, and becomes minimal at the moment the highest level of precision is required: for example, when a table tennis ball is struck (Bootsma \& Van Wieringen, 1991), when a long jumper takes off (Lee et al., 1982), when a somersaulter lands (Bardy \& Laurent, 1998) or, here, when an inter-vehicular gap gap is crossed.

Various factors were expected to modulate the between-trial variability profiles of driving speed and current deviation in our intersection-crossing task. The first factor studied was the time available to prepare crossing, defined by the initial time to arrival (TTA) of the center of the approaching inter-vehicular gap at the intersection. As for tasks of equal difficulty tolerance was hypothesized to be inherently dependent on the time available for adjustment, an increase in TTA was predicted to be accompanied by an increase in initial tolerance. At the beginning of the approach between-trial variability in current deviation was therefore expected be higher for a longer TTA than for a shorter TTA (Figure 1). A second potential mediating factor examined in the current study was the acceleration capability (AC) of the vehicle driven. AC determined the magnitude of the adjustments that could be executed; a vehicle with high acceleration capability allowed the drivers to swiftly modulate their speed. Therefore, for a given TTA, a vehicle with high AC was hypothesized to result in higher initial tolerance, leading us to predict higher between-trial variability in current deviation (Figure 1). As the speed and the size of the traffic gap were identical over all conditions, between-trial variability in current deviation at the time of crossing was expected to be independent of TTA and AC. 
In summary, building on our earlier work on intersection crossing, in this study we more directly tested the hypothesis that control of approach to the intersection was based on a continuous information-movement coupling. To this end, analyses not only focused on average participant behavior but also, for the first time, on between-trial variability in (intermediate adjustment variable) speed and (task-level control variable) current deviation. Markers of information-movement coupling were examined under different conditions of initial tolerance for between-trial variability, operationalized by different initial time-toarrival durations of the traffic gap and different acceleration capabilities of the simulated vehicle driven.

\section{Materials and Methods}

\section{Participants}

Twelve participants, six women and six men $(26.7 \pm 3.8$ years old; $m \pm s d$ ) with normal or corrected to normal vision, volunteered for participation in the experiment. They all held a driver's license for at least three years, with an average duration of $7.5 \pm 2.3$ years. Participants provided written consent prior to the study, which was conducted according to IFSTTAR regulations and the Helsinki Declaration.

\section{Apparatus}

Participants drove a fixed-base SIDROH driving simulator based on a Renault Megane II (4.21 m long, $1.78 \mathrm{~m}$ wide and $1.44 \mathrm{~m}$ high) equipped with a steering wheel, and brake and accelerator pedals. The car was programmed to function with an automatic gearbox so that participants did not have to change gears manually. 


\section{Audio-visual environment}

The audio-visual environment was generated using the ARCHISIM (Espie \& Auberlet, 2007) software package. Using three Epson 485W $60 \mathrm{~Hz}$ projectors, the visual scene was presented on three adjacent screens (1.80-m high by 1.35 -m wide). The left and right screens were oriented inward to maintain a total horizontal visual angle of $150^{\circ}$ for a vertical visual angle of $40^{\circ}$ (see Figure 2a). The virtual environment was also presented in the side and interior rear-view mirrors. The participant's viewpoint was located $1.20 \mathrm{~m}$ from the ground and 2.20 m from the front projection screen. A quadraphonic audio system broadcast sounds related to the car (e.g., engine, starter motor) and other vehicles in the environment.

The simulated environment consisted of a straight textured road, with two lanes for opposing traffic separated by intermittent white lines, running through a flat rural environment (see Figure 2b). The road on which the participant drove (in the right lane) was orthogonally intersected by a similar second road over which a train of vehicles could approach the intersection from the left. Data were collected at a sampling rate of $60 \mathrm{~Hz}$.

\footnotetext{
***** Figure 2 about here $* * * * *$
}

\section{Experimental Design}

Each participant took part in two experimental sessions over which the vehicle's acceleration capability was manipulated. The two sessions took place on the same half-day; there was a 15-minute rest period between the two sessions and the order of presentation of high and low AC was counterbalanced over participants. Each session consisted of a calibration phase and an experimental phase. 


\section{Calibration phase}

The calibration phase preceding an experimental session was designed to familiarize participants with the vehicle's action capabilities, notably its acceleration capability, but also the perceptual consequences of the adjustments produced. It consisted of several tests.

Task. Participants were asked to remain at a constant distance of $30 \pm 9 \mathrm{~m}$ behind a car moving in front of them along a straight road. The speed of the lead car varied. Participants were able to adjust their own speed using the accelerator and brake pedals. The reference distance corresponds to the recommended safe distance between vehicles traveling at a speed of $13.9 \mathrm{~m} / \mathrm{s}(50 \mathrm{~km} / \mathrm{h})$. During the first part of the test, participants received concurrent feedback informing them of their compliance with the instruction (i.e., current inter-vehicular distance $=30 \pm 9 \mathrm{~m})$.

Concurrent feedback was provided by means of a vertical gauge positioned to the left of the central projection screen (Figure 3a). The required distance was represented by a rectangular zone at the center of this gauge (Figure 3b). A real-time cursor, in the form of a sliding horizontal bar, indicated the size of the gap between the lead and participant's cars. When this cursor was within the horizontal rectangle at the center of the gauge (i.e., when the current distance corresponded to the prescribed distance) the gauge was green (Figure 3b). When the cursor was in the upper or lower part of the gauge (i.e., when the current distance was above or below the target distance) it turned red. No numerical information was provided.

In the second part of the test, the gauge disappeared, and the task consisted of maintaining the reference distance without the help of feedback.

Procedure. A calibration trial lasted 145 s (i.e., 2 min and 25 s). At the onset of a trial, participants were stationary, $30 \mathrm{~m}$ behind the car in front of them. The trial began when the 
lead car started to move. This vehicle was programmed to alternate between sections of motion at constant speeds (11 sets) and at variable speeds (11 sets) (Figure 3c). After $65 \mathrm{~s}$ (i.e., during the sixth change in speed of the lead vehicle) concurrent feedback disappeared and the driver had to maintain the reference distance for the next $80 \mathrm{~s}$, despite changes in the speed of the lead vehicle. Each calibration test consisted of one or more block(s) composed of three trials each (see Figure 3c). The magnitude of speed changes imposed by the lead vehicle was indexed to the acceleration capability of the following vehicle (at least during the first two trials in each block). The total driving time of participants, per block, was therefore 7 min 15 s (435 s).

Calibration phase end criterion. Participants had to maintain the prescribed safe distance, initially with feedback and then without. At the end of each trial the percentage of time that the current distance had remained within the prescribed distance during the period without concurrent feedback was calculated, and presented on the central screen. Participants were judged sufficiently "calibrated" when they obtained a score $\geq 80 \%$ in at least two of the three trials of a calibration block. If they did not meet this target, they had to repeat the block. After validation of the calibration phase and a short pause, the experimental phase began.

\section{***** Figure 3 about here $* * * * *$}

\section{Experimental phase (intersection crossing)}

Task. The task was similar to that used in the studies of Louveton et al. (Louveton, Montagne et al., 2012 ; Louveton, Bootsma et al., 2012). Participants were required to safely cross an intersection within a gap delimited by two red cars (2.4 m long, $1.27 \mathrm{~m}$ wide and $1.7 \mathrm{~m}$ high) located in a train of vehicles approaching from their left. This traffic train, composed by four vehicles moving at a constant speed of $10 \mathrm{~m} / \mathrm{s}(36 \mathrm{~km} / \mathrm{h})$, consisted of a truck, the two red cars 
and another truck (see Figure 2b). The gap between the two red cars was $27 \mathrm{~m}$ (i.e., $2.7 \mathrm{~s}$ ). No signage indicating who had priority was present. In the rare cases that a driver collided with another vehicle, a large red triangle appeared on the central screen ${ }^{1}$.

Procedure. At the start of each trial, participants were stationary in the middle of the righthand lane with no other vehicles present in the environment. The trial began when the participant pulled the horizontal lever protruding from the left side of the steering column (normally used for flashing the lights). The participant had to then reach a target speed of 16.0 $\pm 0.55 \mathrm{~m} / \mathrm{s}(57.6 \pm 2.0 \mathrm{~km} / \mathrm{h})$. A procedure equivalent to that used during the calibration phase was used, with a gauge indicating the target speed and current speed positioned in the center of the frontal screen. An 80-m long rectilinear section of road was available for reaching a current speed that corresponded to the prescribed speed (i.e., to bring the slider to the central zone of the gauge). If this requirement was met over the last 20 meters, the gauge disappeared and the actual intersection-crossing trial began, with the train of vehicles appearing on the left. If the participant did not maintain the required speed over the last 20-m stretch, the procedure was repeated. This procedure for standardizing initial participant speed ensured that the train of approaching vehicles was visible at the start of each trial and that (i) the initial participant-intersection time relation and (ii) the initial acceleration capabilities (varying with vehicle speed, see Figure 4a) could be controlled.

\footnotetext{
${ }^{1}$ The intersection-crossing task used in our studies could, at first sight, appear unrealistic, as neither the vehicles in the the traffic train nor the participants ever stopped before crossing. However, several other daily-life driving tasks, such as entering a roundabout or merging into traffic on a motorway, are subject to similar constraints, where negotiating traffic without coming to a full stop may lead to safer behavior. Stopping the car before getting into traffic may indeed prove to be dangerous as the only option is a sudden acceleration, which can dramatically limit the margin of safety.
} 


\section{Independent variables}

Three independent variables were manipulated: The time taken for the train of vehicles to arrive at the intersection (TTA), the car's acceleration capability (AC) and, as in our earlier studies, the initial distance of the participant's car with respect to the intersection (Offset).

Time to Arrival (TTA). The initial distance between the center of the traffic gap and the center of the intersection was manipulated to create different TTAs. Given the constant $10 \mathrm{~m} / \mathrm{s}$ approach speed of the traffic train, initial traffic-train distances from the intersection were set at 65, 75, 85, and $95 \mathrm{~m}$, resulting in TTAs of, respectively, 6.5, 7.5, 8.5, and $9.5 \mathrm{~s}$.

Offset. The initial distance between the participant and the intersection was manipulated to create an offset between their expected time of arrival (if they maintained their $16 \mathrm{~m} / \mathrm{s}$ initial speed) and the time to arrival of the center of the traffic gap at the intersection. For the 6.5, 7.5, 8.5, and 9.5 s TTAs, the starting distance for participants in the No Offset conditions was therefore set at 104, 120, 136, and $152 \mathrm{~m}$, respectively. In these conditions, simply maintaining their initial speed allowed the participant to intercept the center of the intervehicular traffic gap.

In order to induce participants to adjust speed during approach to the intersection, we introduced Early and Late Offsets, as in our earlier studies. In these cases, participants started from an initial distance that was 16 m closer to (Early) or farther from (Late) the intersection with reference to the No Offset condition. Thus, if they continued to move at a constant speed of $16 \mathrm{~m} / \mathrm{s}$, they would arrive at the intersection with an offset of $+1 \mathrm{~s}$ (Early), $0 \mathrm{~s}$ (No Offset), or $-1 \mathrm{~s}$ (Late) with respect to the center of the inter-vehicular gap, irrespective of TTA. Despite this shift, both Early and Late Offsets theoretically allowed the participant to cross the 
intersection without changing speed, as the cars that marked the inter-vehicular traffic gap were separated by a (bumper-to-bumper) interval of $2.7 \mathrm{~s}(27 \mathrm{~m})$.

Acceleration capability (AC). The car's AC was set to either $6.9 \mathrm{~m} / \mathrm{s}^{2}$ or $33.1 \mathrm{~m} / \mathrm{s}^{2}$. Braking capacity remained unchanged $\left(16.9 \mathrm{~m} / \mathrm{s}^{2}\right)$. Both ACs allowed the task to be completed for each offset (see Figure 4b). Beyond the fact that AC manipulations allowed predictions about the time course of between-trial variability of both intermediate and task level variables to be made, they also allowed us to determine to what extent, following Fajen (2005) proposal, perceptual processes and action capabilities are intertwined as part of the control of a goaldirected action.

Combining the four TTAs and the three Offsets gave rise to an experimental block of $4 \times 3=$ 12 trials. The order of presentation of the 12 conditions was randomized within each block. In each of the two AC sessions, participants performed five blocks, for a total of 60 trials per session (i.e., per AC condition) and hence a total of 120 trials for the full experiment.

\footnotetext{
$* * * * *$ Figure 4 about here $* * * * *$
}

\section{Dependent variables}

In order to allow comparison with our earlier work (Louveton, Montagne et al., 2012 ; Louveton, Bootsma et al., 2012 ; Mathieu et al., 2017, Exp. 2), the first part of the analysis examined the average participant behavior under the different experimental conditions for the following variables : gap crossing position and speed and current deviation profiles during the approach phase.

Gap crossing position was defined relative to the time of arrival of the center of the traffic gap at the intersection. A negative crossing time indicated that participants arrived at the 
intersection after the center of the traffic gap (i.e., closer to the trailing vehicle), while a positive value indicated that the participant arrived at the intersection before the center of the gap (i.e., closer to the leading vehicle).

In order to examine the nature of the speed adjustments effected during approach to the intersection, we analyzed the time course of the participant's speed and its effect on future passing position within the traffic gap (current deviation). Current deviation thus captured the instantaneous effect of changes in speed on the future gap crossing position (see Louveton, Montagne et al., 2012 ; Louveton, Bootsma et al., 2012 ; Mathieu et al., 2017), allowing a functional interpretation of observed speed adjustments. Current deviation was initially $+1 \mathrm{~s}$, $0 \mathrm{~s}$ and $-1 \mathrm{~s}$ in the Early, No and Late offset conditions, respectively, and subsequently varied as the participant adjusted speed. Current deviation was calculated every time step, from the time of appearance of the train of traffic until the time the participant crossed the intervehicular traffic gap.

Analyses of speed and current deviation profiles over time were performed at absolute and relative (i.e., normalized) time scales. In both cases a profile discretization procedure was used.

The analysis at an absolute time scale involved a discretization time step of $1 \mathrm{~s}$ for speed and current deviation profiles. To this end, all trials were first synchronized with respect to the moment of participant intersection crossing. Data were then averaged over 1-s periods (60 data points) preceding arrival at the intersection. Since the trial duration differed in each TTA mode, the number of time steps ranged from six (TTA $6.5 \mathrm{~s}$ ) to nine (TTA $9.5 \mathrm{~s}$ ). Even if large differences in the overall duration of the trials prevented us from applying statistical procedures, we deemed useful to report the main characteristics of the regulation behavior exhibited by the participants at the different real time scales. 
The analysis at a relative time scale called upon time normalization and aimed to allow statistical comparisons (by means of ANOVA) of the participant behavior in trials of duration ranging from $6.5 \mathrm{~s}$ to $9.5 \mathrm{~s}$. Following synchronization of all trials with respect to the moment of participant intersection crossing, the normalization process consisted of dividing each trial into four equal time intervals, and calculating average speed and current deviation for the first (Q1), second (Q2), third (Q3) and fourth (Q4) quarters of the approach phase. Hence, the averaging procedure per trial was based on 97, 112, 127 and 142 observations for each quarter in the 6,5, 7,5, 8,5 and 9,5 TTA conditions respectively.

The novel part of the analysis focused on within-participant between-trial variability for speed and current deviation. The discretization procedure described in the previous section allowed, for each variable of interest (i.e., speed and current deviation), to compute 4 mean values for each trial (one per quarter). Between-trial variability corresponded for each quarter to the standard deviation computed over the 5 repetitions. Functional speed adjustment in the approach phase were expected to result in concomitant changes in the (between-trial) variability in speed and current deviation (e.g., Chardenon, Montagne, Buekers \& Montagne, 2002; Camachon, Buekers \& Montagne, 2004). More specifically, they were expected to result in an increase over approach to the intersection in between-trial speed variability, accompanied by a reduction in the between-trial current deviation variability.

\section{Statistical analysis}

Statistical analyses were performed using repeated-measures ANOVAs. The participants' (temporal) position in the gap at the time they reached the intersection was analyzed with the factors Offset (Early, No, Late), TTA (6.5 s, 7.5 s, 8.5 s, 9.5 s) and AC (low, high). For speed and current deviation analyses, four-way ANOVAs were conducted with Offset (Early, No, Late), TTA (6.5 s, 7.5 s, 8.5 s, 9.5 s), AC (low, high) and Time Step (Q1, Q2, Q3 and Q4). 
The same analyses were conducted on within-participant between-trial standard deviations of both current speed and current deviation. When Mauchly's test revealed violations of the sphericity assumption, Greenhouse-Geisser corrections were applied. The statistical significance level was set at $\alpha=.05$. In order to guarantee sufficient power we only considered effects with substantial effect sizes (Cohen, 1988), operationally defined here as $\eta_{p}^{2}>.20$ When appropriate, post hoc analyses were performed using Scheffé tests.

\section{Results}

\section{Calibration Phase}

We first needed to ensure that all participants had integrated the acceleration and deceleration capabilities of their vehicle before beginning the experimental phase. To this end, we examined the number of blocks needed to achieve the required criterion ( $\geq 80 \%$ in at least two of the three trials of a block) as well as performance (percentage time spent at required distance) at the end of the calibration phase. The results indicated that calibration took slightly longer when AC was high $(2.83 \pm 2.2$ blocks and $3.25 \pm 2.7$ blocks for low and high AC, respectively). They also indicated that final performance was comparable over the two ACs (90.5 $\pm 6.7 \%$ and $88.8 \pm 6.2 \%$ for low and high AC, respectively) (see Figures 5a and 5b). These results thus indicated that in each session participants had indeed fully integrated the AC of the vehicle before beginning the experimental phase.

$* * * * *$ Figure 5 about here $* * * * *$

\section{Gap Crossing Position}

An ANOVA on gap crossing position revealed significant main effects for Offset $(F(1.19$, 13.03) $\left.=36.09, p<.001, \eta_{p}^{2}=.77\right)$, AC $\left(F(1,11)=14.21, p<.01, \eta_{p}^{2}=.56\right)$ and TTA 
$\left(F(2.36,25.89)=3.86, p<.05, \eta_{p}^{2}=.26\right)$. None of the interactions reached significance (all $p$ $>.05$, all $\left.\eta_{p}^{2}<.17\right)$.

As can be seen from Figure 6, participants systematically crossed the intersection slightly ahead of the center of the 2.7-s traffic gap (i.e., closer to the leading vehicle than to the trailing vehicle) under all conditions. However, the three experimental variables resulted in subtle, but systematic variations in gap crossing position. The results showed that gap crossing was particularly early (i.e., closer to the leading vehicle) at high AC (on average $+0.41 \mathrm{~s}$ and $+0.26 \mathrm{~s}$ for high and low AC, respectively; Figure 6a), at Early Offset (on average $+0.47,+0.34 \mathrm{~s}$ and $+0.20 \mathrm{~s}$, s for Early, No and Late Offset, respectively; Figure 6b), and at long TTA (+0.37 s compared to +0.31 s for TTA 9.5 s and 6.5 s, respectively; Figure 6c).

$* * * * *$ Figure 6 about here $* * * * *$

Speed and current deviation profiles

\section{$\underline{\text { Absolute time scale }}$}

For reasons of comparison with our earlier studies, the speed and current deviation profiles at the absolute time scale are presented in Figure 7 (left panel). Replicating the findings of Louveton, Montagne et al. (2012), Louveton, Bootsma et al. (2012) and Mathieu et al. (2017, Exp. 2), the three offset conditions give rise to distinct speed profiles. In the present study, such distinct speed profiles were in fact observed under all TTA and AC conditions. Inspection of Figure 7 revealed that speeds differed from very early on in the approach phase and that these differences increased over time. Early Offset resulted in a reduction in speed, followed by an increase when getting closer to the intersection. No Offset resulted in a gradual increase in speed that was amplified closer to the intersection. Late Offset led to a marked early increase in speed, and then another increase closer to the intersection. 
The results also revealed different speed profiles as a function of TTA and AC. Participants took advantage of a vehicle with high AC, and reached a higher speed in the last $2.5 \mathrm{~s}$ before they crossed the intersection. Speed adjustments were more gradual when TTA was long, due to the ability to distribute them over a longer period.

Inspection of the corresponding current deviation profiles in Figure 7 (right panel) indicated that the speed adjustments described above had the effect of allowing participants to target a restricted zone of the gap, located near to the center but slightly closer to the lead vehicle. Globally, profiles showed a gradual reduction in current deviation up to values close to 0 , reflecting the functional nature of speed adjustments, followed by an increase in the final seconds, allowing participants to cross the intersection in the first half of the inter-vehicular traffic interval.

TTA and AC nevertheless also modulated current deviation profiles, which could be differentiated at the beginning of the trial as a function of AC (see Figure 7). Profiles were shifted left when AC was high, reflecting a lower initial speed. While surprising at first glance (the initial speed was imposed), this observation mirrors the fact that speed adjustments occurred very early in the trial (i.e., within the first second). The speed averaging procedure used (cf., method section) allowed such early adjustments to affect the initial speed value. Surprisingly, this was the case regardless of Offset (i.e., even with Early Offset requiring deceleration), although the deceleration capabilities of all vehicles were identical. Conversely, current deviation profiles were far more similar in the second part of the approach.

It is interesting to note that TTA also appeared to play a role; initial drift in current deviation was better tolerated when TTA was long (e.g., 9.5 s) and therefore speed adjustments were more feasible. Conversely, with short TTA (e.g., 6.5 s) initial drift was rather limited as it was more difficult to compensate later. 
The different trial durations did not allow seeking statistical confirmation of the observed effects of Offset, AC and TTA on speed and current deviation profiles at the absolute time scale. We therefore now turn to the time-normalized results.

\section{Relative time scale}

Statistical analysis of the time-normalized data confirmed the observations provided in the previous paragraph.

\section{Speed profiles}

The ANOVA conducted on speed revealed statistically and substantially significant main effects for all four factors: Offset $\left(F(1.14,12.49)=1026.55, p<.001, \eta^{2}=.99\right)$, Time Step $\left(F(1.29,14.16)=45.37, p<.001, \eta_{p}^{2}=.80\right), \operatorname{AC}\left(F(1,11)=14.68, p<.01, \eta_{p}^{2}=.57\right)$ and TTA $\left(F(2.15,23.60)=5.86, p<.01, \eta_{p}^{2}=.35\right)$. It also demonstrated significant first-order interactions for Time Step x Offset $\left(F(3.80,41.76)=166.34, p<.001, \eta_{p}^{2}=.94\right)$, TTA x Offset $\left(F(3.57,39.27)=38.74, p<.001, \eta_{p}^{2}=.78\right)$, AC x Time Step $(F(1.39,15.31)=11.99$, $\left.p<.001, \eta_{p}^{2}=.52\right)$, and Time Step x TTA $\left(F(3.47,38.21)=8.99, p<.001, \eta^{2}=.45\right)$, as well as a significant second-order interactions for Time Step x TTA x Offset $(F(5.86,64.42)=$ 3.87, $\left.p<.001, \eta_{p}^{2}=.26\right)$.

Post-hoc analyses of the first-order interactions confirmed that speed adjustments differed as a function of Offset. Between Q1 and Q3 speed decreased (by $2.58 \mathrm{~m} / \mathrm{s}$ on average, $p<.001$ ), remained unchanged ( $p>.1$ ) and increased (by $2.78 \mathrm{~m} / \mathrm{s}$ on average, $p<.001$ ), in the Early, No and Late Offset conditions, respectively (Figure 8a). Conversely, speed increased systematically (by $3.57 \mathrm{~m} / \mathrm{s}$ on average, $p<.001$ ) between Q3 and Q4 regardless of Offset. 
While AC did not affect adjustments between Q1 and Q3 (all $p>.1$ ), the speed increase produced between Q3 and Q4 was even greater (by $2.16 \mathrm{~m} / \mathrm{s}$ on average) for high AC (all $p<$ .001), especially when TTA was short (Figure 8b).

The post-hoc analysis of the Time Step x TTA x Offset interaction confirmed that speed adjustments during the approach due to different Offsets were largely comparable regardless of TTA. Only Late Offset resulted in a higher final (Q4) speed $(+1.88 \mathrm{~m} / \mathrm{s}$ and $+2.21 \mathrm{~m} / \mathrm{s})$ for a short TTA (i.e., $6.5 \mathrm{~s})$ than for the longest TTAs (8.5 s and $9.5 \mathrm{~s})(p<.001)$ (Figure 8c).

$* * * * *$ Figure 8 about here $* * * * *$

\section{Current deviation}

The ANOVA on current deviation revealed statistically and substantially significant main effects for Offset $\left(F(1.71,18.76)=263.35, p<.001, \eta^{2}{ }_{p}=.96\right)$, Time Step $(F(1.71,18.81)=$ $\left.16.01, p<.001, \eta_{p}^{2}=.59\right)$ and $\mathrm{AC}\left(F(1,11)=7.57, p<.05, \eta_{p}^{2}=.41\right)$. The main effect of TTA was not significant $\left(p>.38, \eta_{p}^{2}=.09\right)$. The analysis also showed first-order interactions for Time Step x Offset $\left(F(3.77,41.46)=209.61, p<.001, \eta_{p}^{2}=.95\right)$, AC x Time Step $(F(2.02$, $\left.22.17)=4.95, p<.01, \eta_{p}^{2}=.31\right)$ and Time Step $\mathrm{x} \operatorname{TTA}\left(F(2.57,28.28)=4.40, p<.001, \eta_{p}^{2}=\right.$ $.29)$.

Post hoc analysis of the first-order interactions showed that current deviation profiles were different in the three Offset modalities (Figure 9). Between Q1 and Q3, current deviation decreased by $0.89 \mathrm{~s}(p<.001)$, remained constant $(p>.1)$ and decreased by $0.74 \mathrm{~s}(p<.001)$ in Early, No and Late Offset conditions respectively, once again reflecting the functional nature of speed adjustments. Conversely, current deviation increased indiscriminately (i.e., regardless of offset) in Q4. This pattern was modulated by AC. When AC was high, current deviation was higher in Q2, under Late and No Offset conditions $(0.40 \mathrm{~s} ; p<.001)$, and the 
increase in current deviation between Q3 and Q4 was higher (0.48 s; $p<.001)$. Both results reflect the participant's ability to harness the power of the vehicle, to the extent that their lateness in Q2 could be offset by a subsequent acceleration. Similarly, a final high acceleration allowed participants to cross the inter-vehicular traffic gap a little more safely.

***** Figure 9 about here $* * * * *$

\section{Variability of speed}

The ANOVA on between-trial variability of speed revealed statistically and substantially significant main effects for $\mathrm{AC}\left(F(1,11)=69.65, p<.001, \eta_{p}^{2}=.86\right)$, Time Step $\left(F(2.45,26.97)=57.46, p<.001, \eta_{p}^{2}=.84\right)$, Offset $\left(F(1.77,19.52)=3.81, p<.05, \eta_{p}^{2}=.26\right)$ and TTA $\left(F(2.23,24.56)=3.07, p<.05, \eta_{p}^{2}=.22\right)$. It also highlighted significant interactions for Offset $\mathrm{x}$ Time Step $\left(F(2.53,27.86)=6.67, p<.001, \eta_{p}^{2}=.38\right)$ and AC $\mathrm{x}$ Time Step $\left(F(2.11,23.24)=4.19, p<.05, \eta_{p}^{2}=.28\right)$.

Post-hoc analysis of the AC x Time Step interaction indicated that, for both ACs, the standard deviation of current speed increased between Q1 and Q2 $(0.46 \mathrm{~m} / \mathrm{s} ; p<.01)$ and then stabilized between Q2 and Q3 ( $p>$.1) (Figure 10a). Conversely, it only increased between Q3 and Q4 when AC was high $(0.40 \mathrm{~m} / \mathrm{s} ; p<.001)$. Finally, we observed that speed variability was higher by $+0.61 \mathrm{~m} / \mathrm{s}$ when AC was high $(p<.01)$.

Post-hoc analysis of the Offset $\mathrm{x}$ Time Step interaction indicated an increase in speed variability between Q1 and Q2 and between Q3 and Q4 in Early and No Offset conditions ( $p$ $<.01)$ (Figure 10b). In the Late Offset condition, it increased between Q1 and Q3 $(p<.05)$.

***** Figure 10 about here $* * * * *$ 


\section{Variability of current deviation}

The ANOVA on between-trial variability in current deviation revealed statistically and substantially significant main effects for $\operatorname{AC}\left(F(1,11)=91.94, p<.001, \eta^{2} p=.89\right)$, Time Step $\left(F(2.02,22.18)=41.31, p<.001, \eta_{p}^{2}=.79\right)$ and TTA $\left(F(2.55,28.02)=29.00, p<.001, \eta_{p}^{2}=\right.$ .72). The substantial main effect of Offset $\left(F(1.67,18.39)=3.43, p=.051, \eta_{p}^{2}=.24\right)$ came close to the statistical significance level. The analysis also revealed significant first-order interactions for AC x Time Step $\left(F(2.19,24.11)=10.39, p<.001, \eta_{p}^{2}=.49\right)$, TTA $x$ Time Step $\left(F(4.28,47.12)=5.09, p<.001, \eta_{p}^{2}=.32\right)$ and AC x TTA $(F(2.41,47.12)=3.95, p<$ $\left..05, \eta_{p}^{2}=.26\right)$.

Analysis of the AC x Time Step interaction (Figure 11a) indicated that the variability in current deviation was higher when AC was high in Q1 to Q3 (0.23 s on average; $p<.001)$, but the final variability (Q4) was similar regardless of AC ( $p>$.99). Furthermore, the pattern of change was the same for both ACs, with an increase from Q1 to Q2 (average increase of 0.20 s; $p<.01)$, stabilization between Q2 and Q3 ( $p>$.1) and a decrease between Q3 and Q4 (mean decrease of $0.24 \mathrm{~s} ; p<.01$ ).

Post-hoc analysis of the TTA x Time Step interaction (Figure 11b.) indicated globally equivalent patterns of change for all TTA's: increased variability between Q1 and Q2, stabilization between Q2 and Q3, and a decrease between Q3 and Q4. However, in Q2 variability was lower for TTA $6.5 \mathrm{~s}$ than for $8.5 \mathrm{~s}$ and $9.5 \mathrm{~s}$ (differences of $0.24 \mathrm{~s}$ and $0.30 \mathrm{~s}$, respectively, $p<.001)$. In other time intervals (Q1, Q3 and Q4) the variability in current deviation did not differ as a function of TTA.

Post-hoc analysis of the AC x TTA interaction (Figure 11c) indicated that the variability in current deviation was higher when AC was high $(0.18 \mathrm{~s}$ on average; $p<0.5)$ for all four TTA. 
Moreover, with low AC, it was lower for TTA $6.5 \mathrm{~s}$ than for $9.5 \mathrm{~s}$ (a difference of $0.12 \mathrm{~s} ; \mathrm{p}<$ .05). With high AC, it was lower for TTA $6.5 \mathrm{~s}$ than for $8.5 \mathrm{~s}$ and $9.5 \mathrm{~s}$ (a difference of $0.20 \mathrm{~s}$ and 0.24 s, respectively, $p<.001)$.

***** Figure 11 about here $* * * * *$

\section{Discussion}

In this study we analyzed the adjustments (i.e., speed modulations) efffected by drivers approaching and crossing an intersection with an incoming flow of traffic. Specifically, the goal was to identify specific markers of information-movement coupling, by examining the participants' car kinematics during the approach phase up to intersection crossing. The implementation of such a coupling was not only hypothesized to result in a gradual gain in average accuracy during the approach (as already observed in earlier studies of intersection crossing), but also to give rise to opposing changes in between-trial variability in driving speed and current deviation. Indeed, the (between-trial) variability in (intermediate adjustment variable) speed was predicted to increase during the approach, while the (between-trial) variability in (task-level control variable) current deviation was predicted to concomittantly decrease. We also hypothesized that these effects would be mediated by the time allowed to perform the task (TTA) and the acceleration capabilities (AC) of the vehicle.

Improved accuracy. Our results confirmed and extended the results obtained in our previous work (Louveton, Bootsma et al., 2012; Louveton, Montagne et al., 2012; Mathieu et al., 2017, Exp. 2). They once again demonstrated that participants target a relatively restricted zone in the inter-vehicular traffic gap, located slightly before its center. As highlighted in the introduction section, targeting such a zone preserves a large margin of safety and allows sufficient time to cross the intersection (see Chihak et al., 2010, 2014 for similar results). The 
robustness of this result was clearly confirmed in the present study, exploring the effects of the time available until crossing (with initial traffic gap TTAs ranging from $6.5 \mathrm{~s}$ to $9.5 \mathrm{~s}$ ) and the acceleration capabilities (AC) of the vehicle driven. While task factors such as TTA and AC did affect the average position within the traffic gap where intersection is crossed to a certain extent, the effect was relatively small (mean gap crossing positions of $+0.26 \mathrm{~s}$ vs +0.41 $\mathrm{s}$ for low and high AC, respectively, and $+0.31 \mathrm{~s}$ vs $+0.37 \mathrm{~s}$ for TTAs of $6.5 \mathrm{~s}$ and $9.5 \mathrm{~s}$, respectively). Thus, despite the considerable differences in task conditions created by manipulation of the two factors, participants always passed the intersection in a rather limited zone within the traffic gap. These results were consistent with our previous findings (Louveton, Bootsma et al., 2012; Louveton, Montagne et al., 2012; Mathieu et al., 2017). Moreover, speed adjustments were once again found to be initiated early on and distributed throughout the approach phase. This became even clearer in the present contribution, with the exploration of driver behavior under conditions of different approach durations (i.e., TTA's). Interestingly, the distribution of speed adjustments over the available time span also resulted in driving behavior (i.e., speed profiles) that was largely independent of vehicle's acceleration capabilities during a large portion of the approach. Indeed, acceleration-capability related differences only came to the fore when in the last seconds before crossing participants abruptly accelerated. This typical final acceleration, also observed in our earlier studies and in the studies of Chihak, Plummert and colleagues (Chihak et al., 2010, 2014), appears to occur when participants have secured crossing within the traffic gap and might serve maximize the safety margin by minimizing the time needed to cross the intersection.

Current deviation profiles confirmed that speed adjustments aimed to improve accuracy of the future position position within the traffic gap. Overall, the results demonstrated a progressive reduction in current deviation (i.e., an improvement in accuracy) during the approach to the intersection. The increase in current deviation in the last seconds before crossing is the result 
of the abrupt final acceleration discussed above, targeting the identified comfort zone. Once again, the comparative analysis of current deviation profiles observed in different experimental conditions brought out both strong global similarities and some specific features reflecting experimental constraints (TTA and AC). When AC was high, participants seemed to tolerate a drift in current deviation at the beginning of the approach, commensurate with the

ample opportunity to regulate their speed later. Conversely, limited AC led them to restrict this drift, in order to be able to make the speed adjustments necessary to carry out the task. Similar results were observed when TTA was manipulated; initial drift in current deviation was larger when TTA is longer, with longer TTA of course implying more time to make the necessary speed adjustments.

Change in variability patterns. In the present contribution we not only considered average participant behavior, as in our earlier studies on intersection crossing, but also focused on patterns of (within-participant between-trial) variability in the intermediate adjustment variable speed and the task level control variable current deviation. This novel analysis brought out a gradual increase over time in the variability of speed, while variability of current deviation evolved according to inverted U-like profile. In order to understand the latter profile, it is important to bear in mind that our procedure standardized both speed and current deviation at the outset of each trial. The initial (Q1) values of the between-trial variability in speed and current deviation were thus necessarily small and underestimated with respect to natural situations. Consequently, for the present purposes we focused on the subsequent change (Q2 to Q4) in variability patterns. Consistent with our predictions, variability profiles revealed opposing characteristics; a progressive increase in the variability of speed was accompanied by a progressive reduction in the variability of current deviation that reached its lowest value in the last time interval before the crossing. 
This pattern of results further reinforces the conclusion that speed adjustments (leading to increased variability) are functional, serving to target a specific zone in the traffic gap (leading to low variability in current deviation). The adjustments described here in the framework of intersection crossing tasks are in all respects consistent with those described in the context of other tasks that necessitate the production of goal-directed movements (e.g., Lee et al., 1982; Bootsma \& Van Wieringen, 1991; Bardy \& Laurent, 1998; Camachon, Buekers \& Montagne, 2004; Chardenon, Montagne, Buekers \& Laurent, 2002). Most importantly, the gradual reduction in the variability of the task-level control variable (i.e., funnel-like type of control) is a marker of control based on information-movement coupling.

The variability analysis also provided a tool to understanding how TTA and AC influenced the speed adjustments produced by participants and affected overall variability, without altering its time profile. Under all experimental conditions, a concomitant and opposite change in variabilities of speed and current deviation was observed. In agreement with our hypotheses, the magnitude of variability prior to crossing was larger in higher AC and longer TTA conditions. Yet, variability in current deviation was always lowest at the very last moment before crossing. This latter result indicated that the operative conditions for task completion (here TTA and AC) modulated the tolerance associated with the implementation of the information-movement cycle. This tolerance was higher when adjustments could be distributed over a longer time time, but also when AC allowed rapid speed adjustments.

Implications for the identification of the underlying control mechanisms. Taken together, the results are fully consistent with the implementation of a control based on informationmovement coupling. Speed adjustments gradually improve movement accuracy, in such a way that the inter-vehicular traffic gap can be crossed safety. A flexible control mechanism is implemented, which can adapt the regulation behavior to the specific conditions that are 
encountered. Not only is this mechanism based on the consideration of an informational support that allows the agent to access, at any time, the state of their relationship with the environment, but task-related variables such as TTA and AC also modulate the effect of the coupling and consequently the adjustments that are produced.

Further studies will be necessary to identify precisely the perceptual substrate involved in this coupling. In order to test the contribution of potential information sources, such as the (rate of change of) optical size of traffic train vehicles or their bearing with respect to the driver's lane, such information sources could be manipulated over the course of a trial. The observed effects on speed regulation would help to move towards the formalization of a law of control.

We believe our results also provide a useful complement to recent studies that put action capabilities at the heart of the control process in goal-directed action (e.g., Fajen, 2005, 2007; Morice, Diaz, Fajen, Basilio \& Montagne, 2015; Marti, Morice \& Montagne, 2015). A goal of studies that aim to formalize control laws could therefore be to consider the capacity for action in the operationalization of the information-movement relation.

From a more applied point of view, our results could prove useful when designing adaptive driver assistance systems (ADAS) for use at intersections. While the majority of the ADAS tested so far provided the drivers with cues related to the environment (e.g., the speed of approaching vehicles; Houtenbos et al., 2017), more powerful visual enhancements, i.e., related to the state of the agent-environment system, could benefit the driver. We already tested the usefulness of this type of visual enhancements in the context of landing an airplane (Huet, Jacobs, Camachon, Goulon \& Montagne, 2009; Huet, Jacobs, Camachon, Missenard, Gray \& Montagne, 2011). As a follow-up of these studies, the usefulness of ADAS providing the driver with an information related to the current deviation would certainly be worth considering. 


\section{Acknowledgements}

We wish to thank F. Vienne and I. Aillerie (IFSTTAR, COSYS-LEPSIS) for designing the displays. 


\section{References}

Bardy, B. G., \& Laurent, M. (1998). How is body orientation controlled during somersaulting? Journal of Experimental Psychology: Human Perception and Performance, 24, 963-977.

Bootsma, R. J. (1998). Ecological movement principles and how much information matters. In A. A. Post, J. R. Pijpers, P Bosch, \& M. S. J. Boschker (Eds.), Models in Human Movement Science (pp. 51-63). Enschede: PrintPartners Ipskamp.

Bootsma, R. J., \& Van Wieringen, P. C. W. (1990). Timing an attacking forehand drive in table tennis. Journal of Experimental Psychology: Human Perception and Performance, $16,21-29$.

Bootsma, R. J., Houbiers, M. H. J., Whiting, H. T. A., \& Van Wieringen, P. C. W. (1991). Acquiring an attacking forehand drive: the effects of static and dynamic environmental conditions. Research Quarterly for Exercise and Sport, 62, 276-284.

Camachon, C., Buekers, M. J., \& Montagne, G. (2004). Is the learning of goal-directed displacement effector independent? Human Movement Science, 23, 239-255.

Chardenon, A., Montagne, G., Buekers, M. J., \& Laurent, M. (2002). The visual control of ball interception during human locomotion. Neuroscience Letters, 334, 13-16.

Chardenon, A., Montagne, G., Laurent, M., \& Bootsma, R. J. (2004). The perceptual control of goal-directed locomotion: a common architecture for interception and navigation? Experimental Brain Research, 158, 100-108. 
Chihak, B. J., Plumert, J. M., Ziemer, C. J., Babu, S., Grechkin, T., Cremer, J. F., \& Kearney, J. K. (2010). Synchronizing self and object movement: How child and adult cyclists intercept moving gaps in a virtual environment. Journal of Experimental Psychology: Human Perception and Performance, 36, 1535-1552.

Chihak, B. J., Grechkin, T. Y., Kearney, J. K., Cremer, J. F., \& Plumert, J. M. (2014). How children and adults learn to intercept moving gaps. Journal of Experimental Child Psychology, 122, 134-152.

Cohen, J. (1988). Statistical Power Analysis for the Behavioral Sciences, 2nd Ed. Hillsdale NJ: Erlbaum.

Espie, S., \& Auberlet, J. M. (2007). ARCHISIM: a behavioral multi-actors traffic simulation model for the study of a traffic system including ITS aspects. International Journal of ITS Research, 5, 7-16.

Fajen, B. R. (2005). Perceiving possibilities for action: On the necessity of calibration and perceptual learning for the visual guidance of action. Perception, 34, 717-740.

Fajen, B. R. (2007). Affordance-based control of visually guided action. Ecological Psychology, 19(4), 383-410.

Gibson, J. J. (1986). The ecological approach to visual perception. Hillsdale, NJ: Lawrence Erlbaum Associates, Inc. (Original work published in 1979)

Houtenbos, M., de Winter, J. C. F., Hale, A. R., Wieringa, P. A., \& Hagenzieker, M. P. (2017). Concurrent audio-visual feedback for supporting drivers at intersections : a study using two linked driving simulators. Applied Ergonomics, 30-42. 
Huet, M., Jacobs D.M., Camachon, C., Goulon, C., \& Montagne. G. (2009). Self-controlled concurrent feedback facilitates the learning of the final approach phase in a fixed-base flight simulator. Human factors, 51, 858-871.

Huet, M., Jacobs, D.M., Camachon, C., Missenard, O., Gray, R., \& Montagne, G. (2011). The Education of Attention as Explanation of Variability-of-Practice Effects: Learning the Final Approach Phase in a Flight Simulator. Journal of Experimental Psychology: Human Perception and Performance, 37, 1841-54.

Lee, D. N., Lishman, J. R., \& Thomson, J. A. (1982). Regulation of gait in long jumping. Journal of Experimental Psychology: Human Perception and Performance, 8, 448458.

Louveton, N., Bootsma, R. J., Guerrin, P., Berhelon, C., \& Montagne, G. (2012a). Intersection crossing considered as intercepting a moving traffic gap: effects of task and environmental constraints. Acta Psychologica, 141, 287-294.

Louveton, N., Montagne, G., Berthelon, C., \& Bootsma, R. J. (2012b). Intercepting a moving traffic gap while avoiding collision with lead and trail vehicles: Gap-related and boundary-related influences on drivers' speed regulations during approach to an intersection. Human Movement Science, 31, 1500-1516.

Marti, G., Morice, A. H. P., \& Montagne, G. (2015). Drivers’ decision-making when attempting to cross an intersection results from choice between affordances. Frontiers in Human Neuroscience, 9; 8:1026.

Mathieu, J., Bootsma, R. J., Berthelon, C., \& Montagne, G. (2017). Judging arrival times of incoming traffic vehicles is not a prerequisite for safely crossing an intersection: 
Differential effects of vehicle size and type in passive judgment and active driving tasks. Acta Psychologica, 173, 1-12.

McLeod, R. W., \& Ross, H. E. (1983). Optic flow and cognitive factors in time-to-collision estimates. Perception, 12, 417-423.

Montagne, G., Laurent, M., Durey, A., \& Bootsma, R. J. (1999). Movement reversals in ballcatching. Experimental Brain Research, 129, 87-92.

Montagne, G., Cornus, S., Glize, D., Quaine, F., \& Laurent, M. (2000). A 'perception-action coupling' type of control in long-jumping. Journal of Motor Behavior, 32, 37-44.

Morice, A. H. P., Diaz, G. J., Fajen, B. R., Basilio, N., \& Montagne, G. (2015) An affordancebased approach to visually guided overtaking, Ecological Psychology, 27, 1-25.

Schiff, W., \& Detwiler, M. (1979). Information used in judging impending collisions. Perception, 8, 647-658.

Scott, M. A., Li, F-X., \& Davids, K. (1997). Expertise and the regulation of gait in the long jump approach phase. Journal of Sport Sciences, 15, 597-605.

Warren, W. H., Young, D. S., \& Lee, D. N. (1986). Visual control of step length during running over irregular terrain. Journal of Experimental Psychology: Human Perception and Performance, 12, 259-266.

Warren, W. H. (1988). Action mode and laws of control for the visual guidance of action. In O. Meijer \& K. Roth (Eds.), Complex movement behavior: 'the' motor-action controversy (pp. 339-380). Amsterdam: North-Holland. 
Warren, W. H., Kay, B. A., Zosh, W. D., Duchon, A. P., \& Sahuc, S. (2001). Optic flow is used to control human walking. Nature Neuroscience, 4, 213-216.

Warren, W.H. (2006) The dynamics of perception and action. Psychological Review, 113, 358-389. 


\section{Figure Captions}

Figure 1: Schematic representation of the change in temporal tolerance as a function of the time before the arrival of the line of vehicles (TTA) and the vehicle's response capacities.

Figure 2: An exterior view of the driving simulator with the three projectors and screens (a); the participant's view from inside the simulator (b). The scene is presented on the three screens and in the side and rear-view mirrors. Here, the participant could see two trucks separated by two red cars with a 27-m gap between them (b).

Figure 3: Description of the calibration phase during which participants had to maintain a constant inter-vehicular distance, despite changes in the speed of the lead vehicle (a) At the beginning of each test, subjects were provided with concurrent feedback in the form of a gauge positioned on the left of the screen (b) showing the difference between the prescribed distance and the current distance. Each calibration block comprised three trials in which the speed of the lead vehicle varied more or less abruptly as a function of whether participants were driving a vehicle with a high acceleration capacity (black line) or low acceleration capacity (blue line) (c). During each trial, participants were initially provided with concurrent feedback (the shaded area), which disappeared in the second half of the trial.

Figure 4: Evolution over time of the maximum acceleration that can be produced by the two types of vehicles (a) and crossing places with reference to the inter-vehicular interval with maximum acceleration (b). These crossing locations are shown for each type of vehicle, in Late offset conditions and for the four arrival time conditions in the inter-vehicular temporal gap. The red rectangles represent the two cars that delimit the inter-vehicular temporal gap.

Figure 5: Illustrations of the regulation behaviour produced at the end of the calibration phase by a participant driving a vehicle with high acceleration (a) and low acceleration (b). The grey 
background represents the period during which participants were provided with concurrent feedback.

Figure 6: Gap crossing position as a function of acceleration capability (a), offset (b) and Time to Arrival (c). ${ }^{* * *} p<0.001$ and ${ }^{* *} p<0.01$ significant differences. Error bars indicate inter-participant standard deviation.

Figure 7: Average current speed (left panel) and average current deviation (right panel) as a function of the absolute time to reach the intersection. Error bars indicate inter-participant standard deviation.

Figure 8: Average current speed as a function of relative time for AC, Time Step and Offset (a), AC, TTA and Time Step (b) and Offset, TTA and Time Step (c). Error bars indicate interparticipant standard deviation.

Figure 9: Average current deviation for Time Step, AC and Offset. Error bars indicate interparticipant standard deviation.

Figure 10: Average intra-participant current speed variability for Time Step and AC (a) and Offset and Time Step (b). Error bars indicate inter-participant standard deviation.

Figure 11: Average intra-participant standard deviation of current deviation for AC and Time Step (a), TTA and Time Step (b) and AC and TTA (c). Error bars indicate inter-participant standard deviation. 
Figure 1

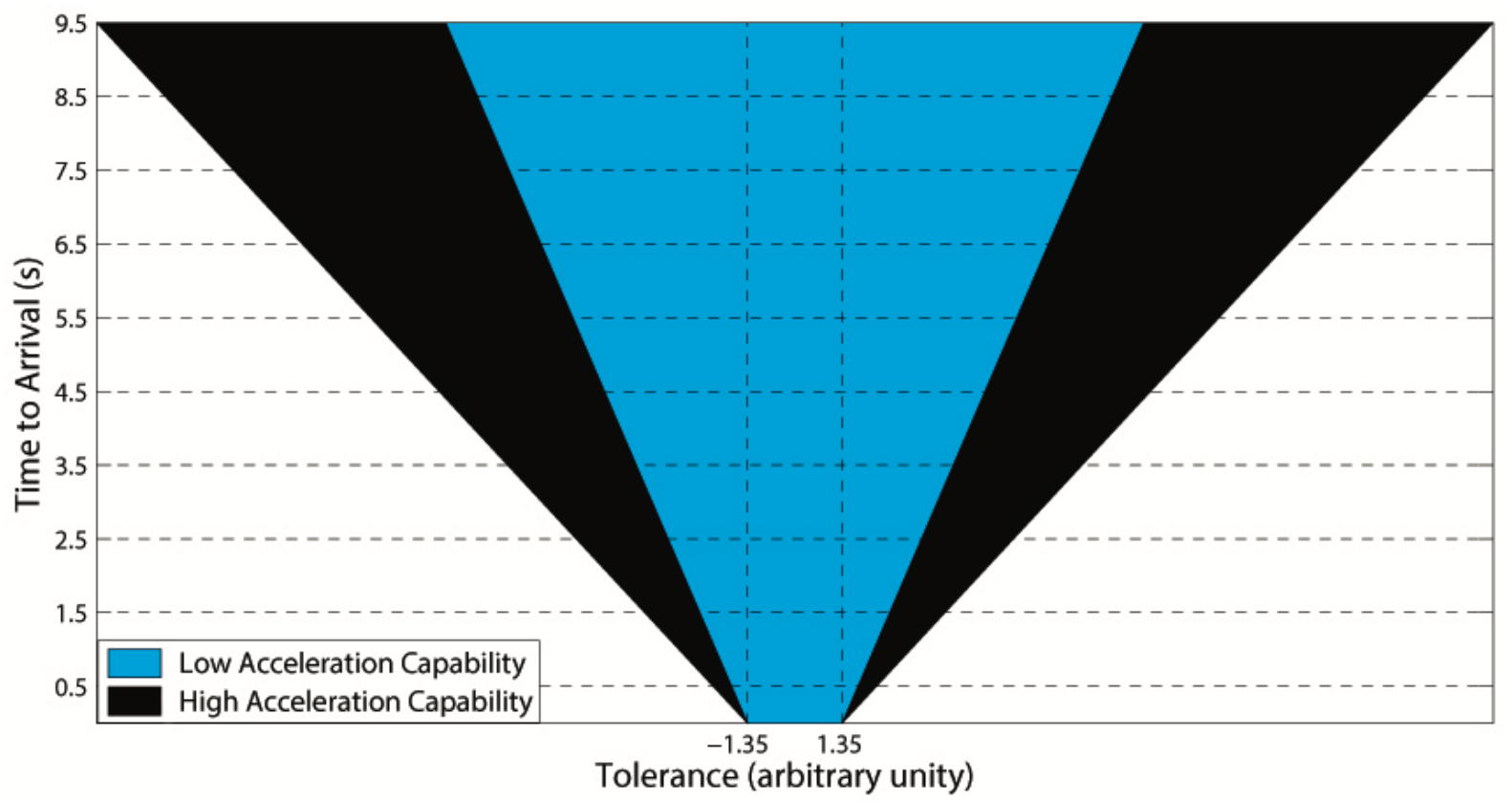


Figure 2

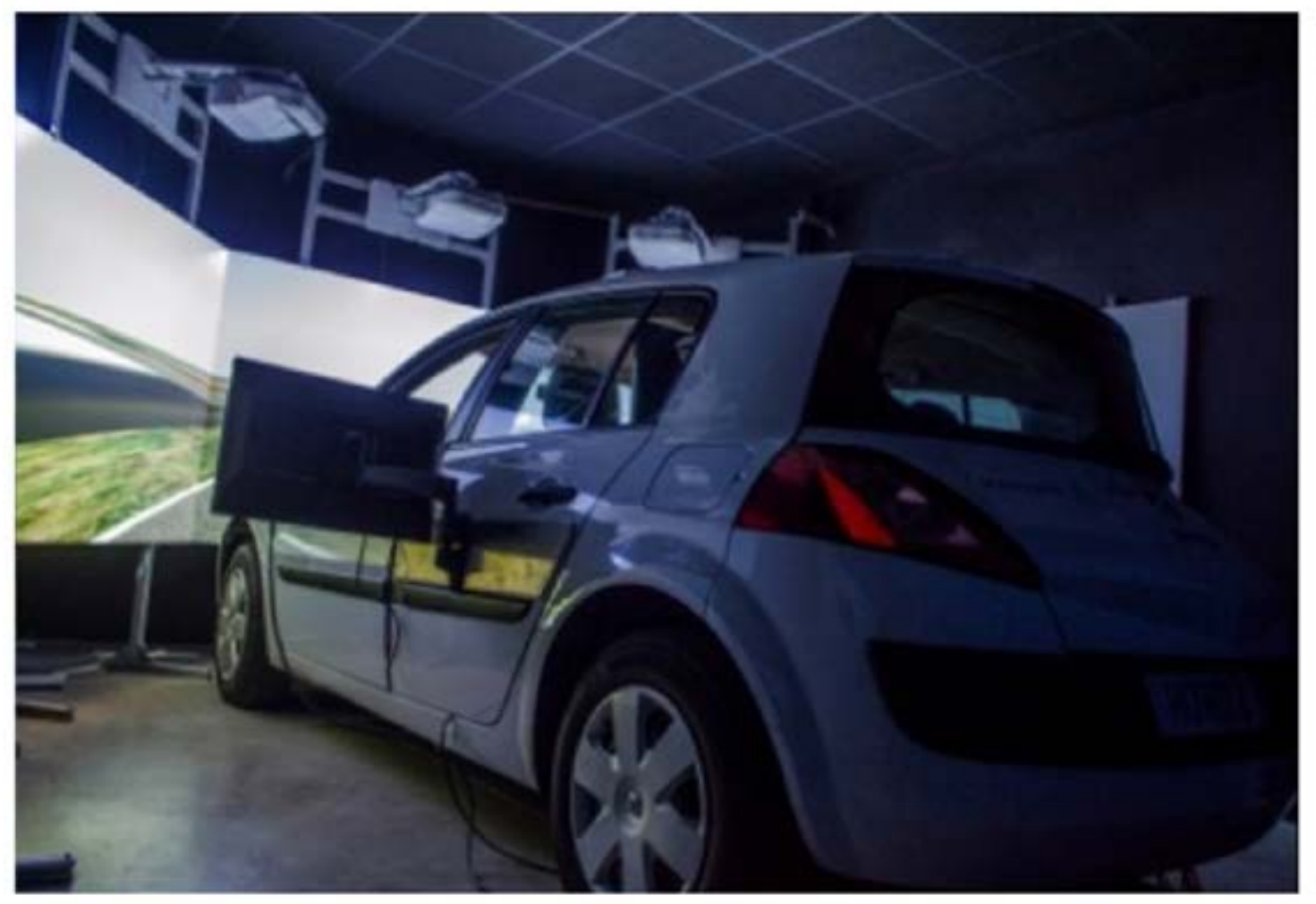

(s

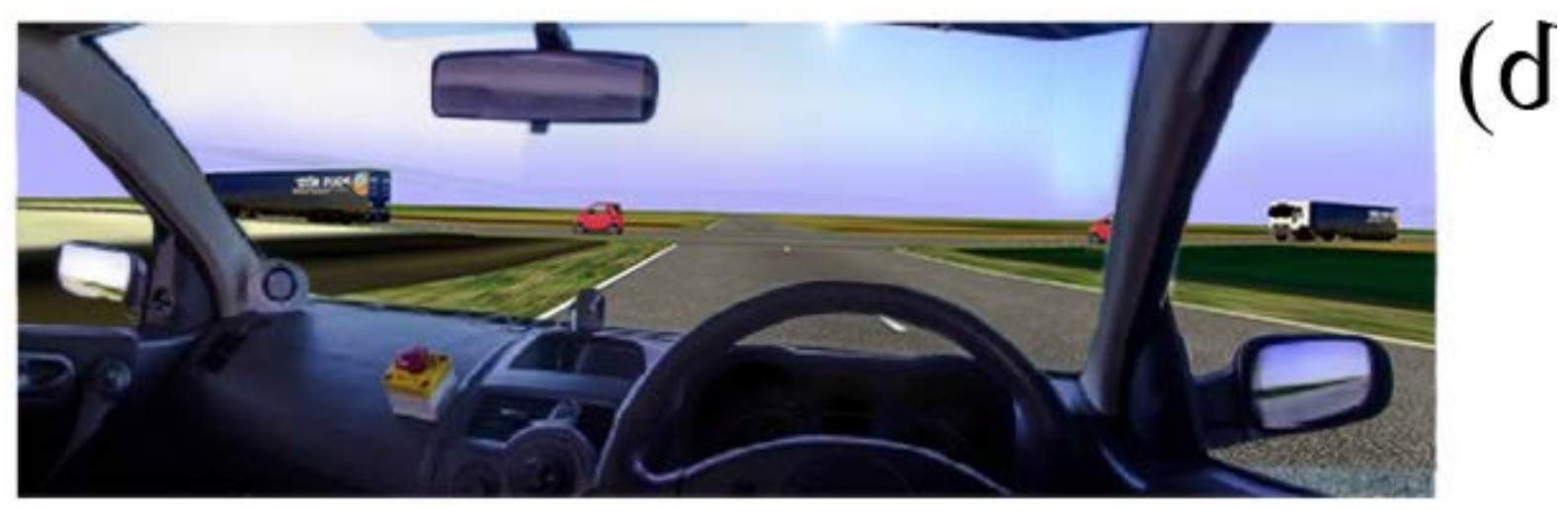


Figure 3

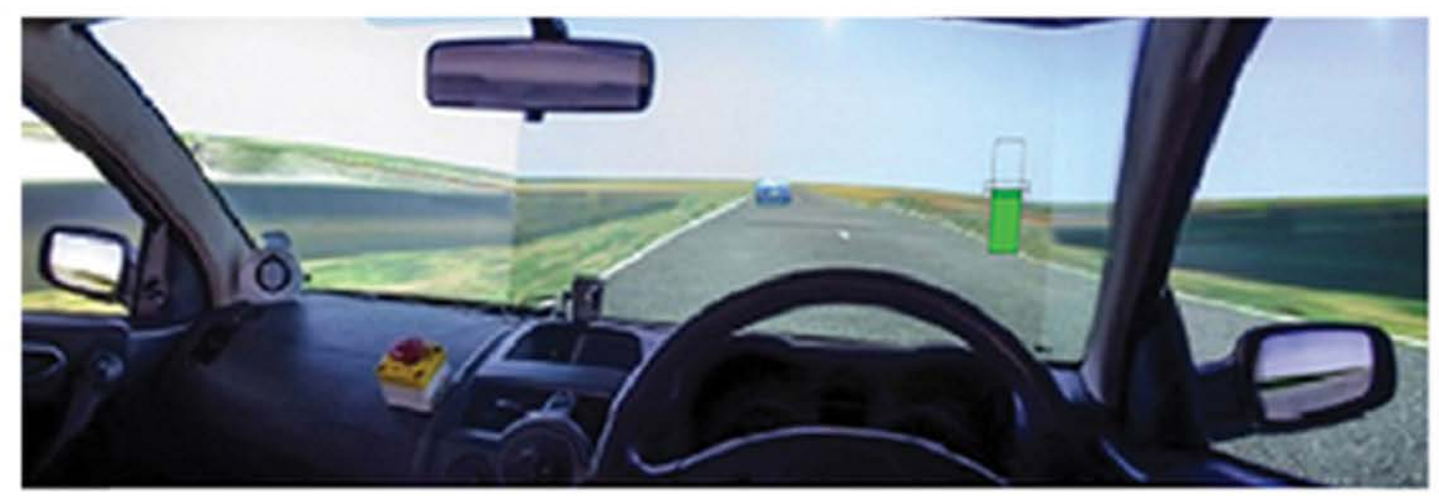

(s
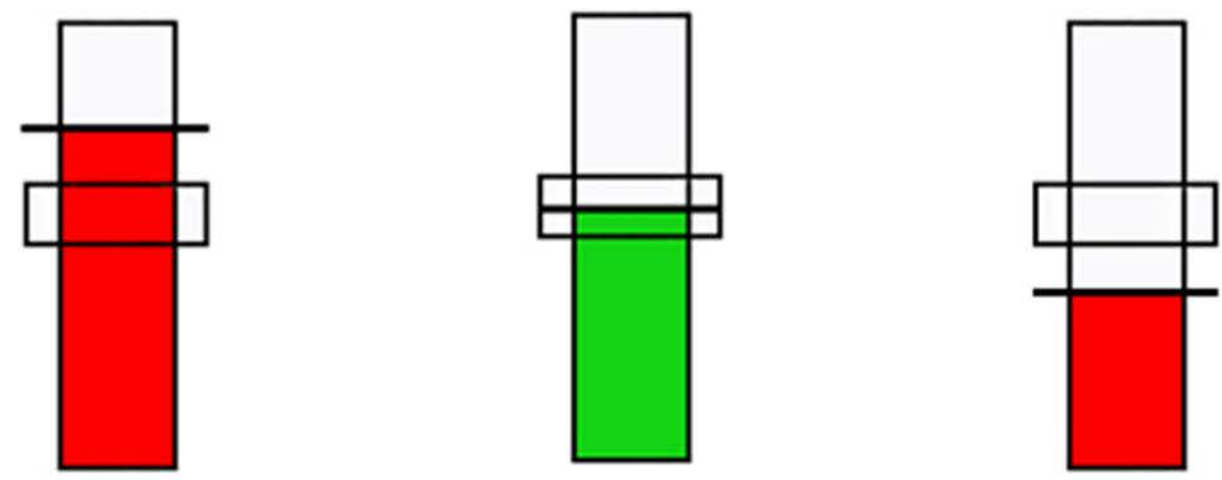

(d

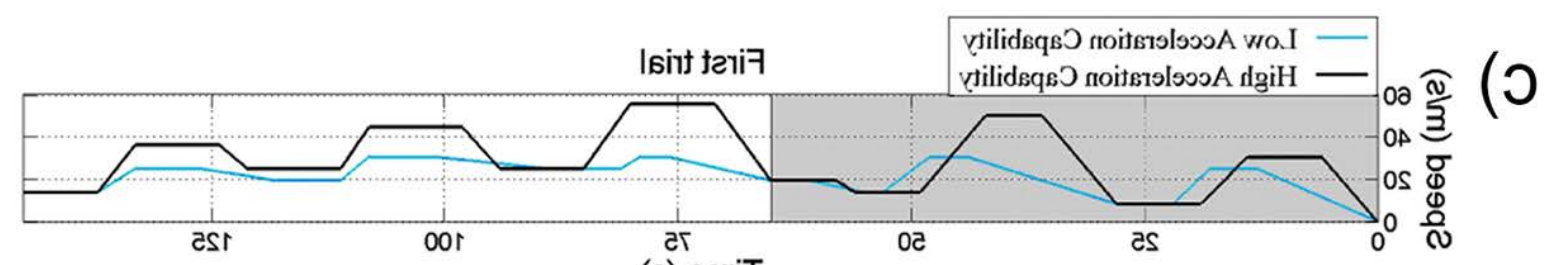

(2) जmiT

Isint brooงอ

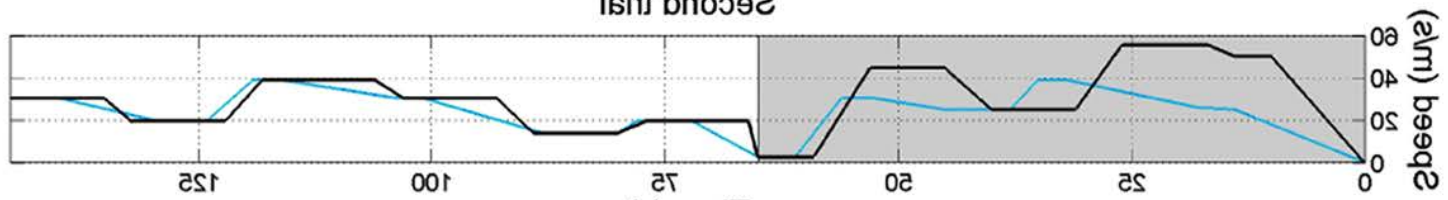

(c) эmiT

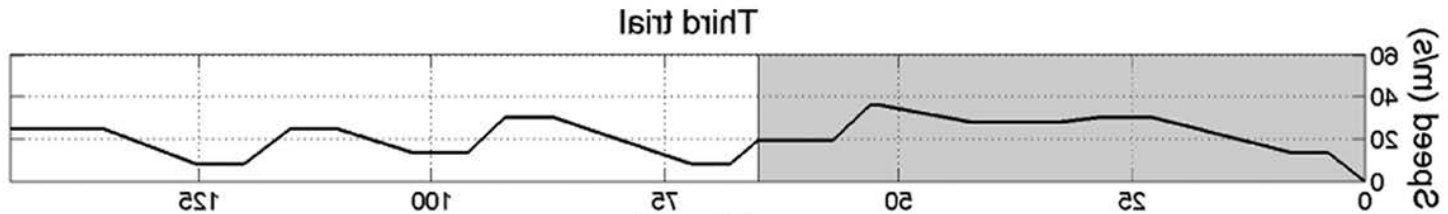

(a) өmiT 
Figure 4
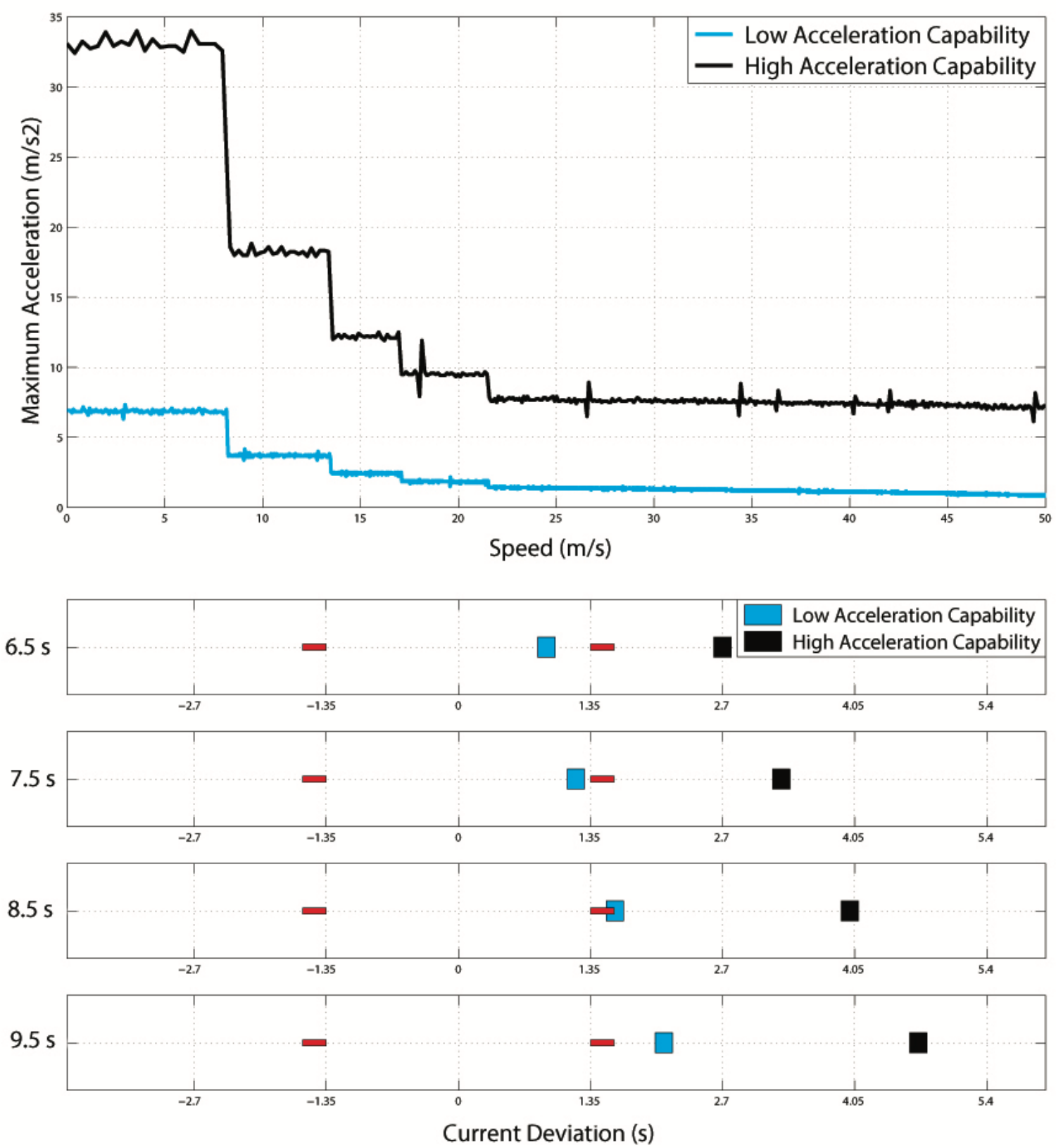
Figure 5

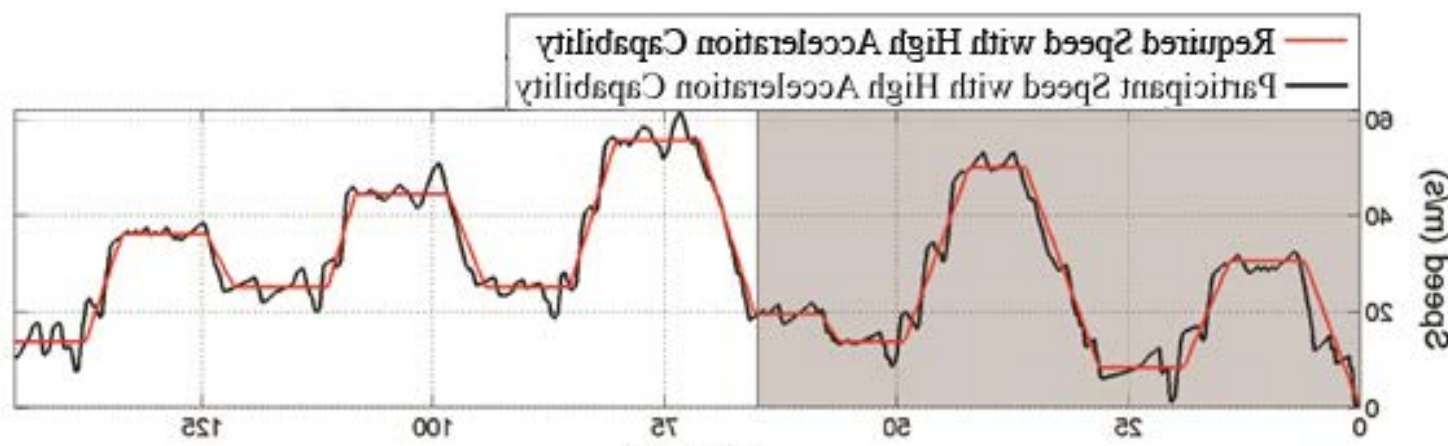

(2) эmiT

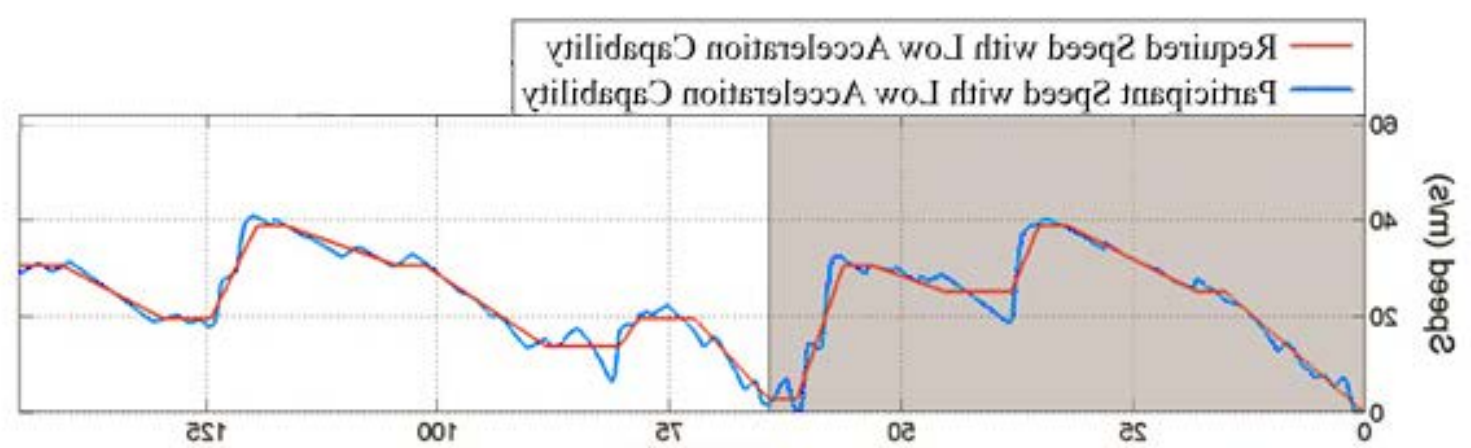

(c) 9miT 
Figure 6

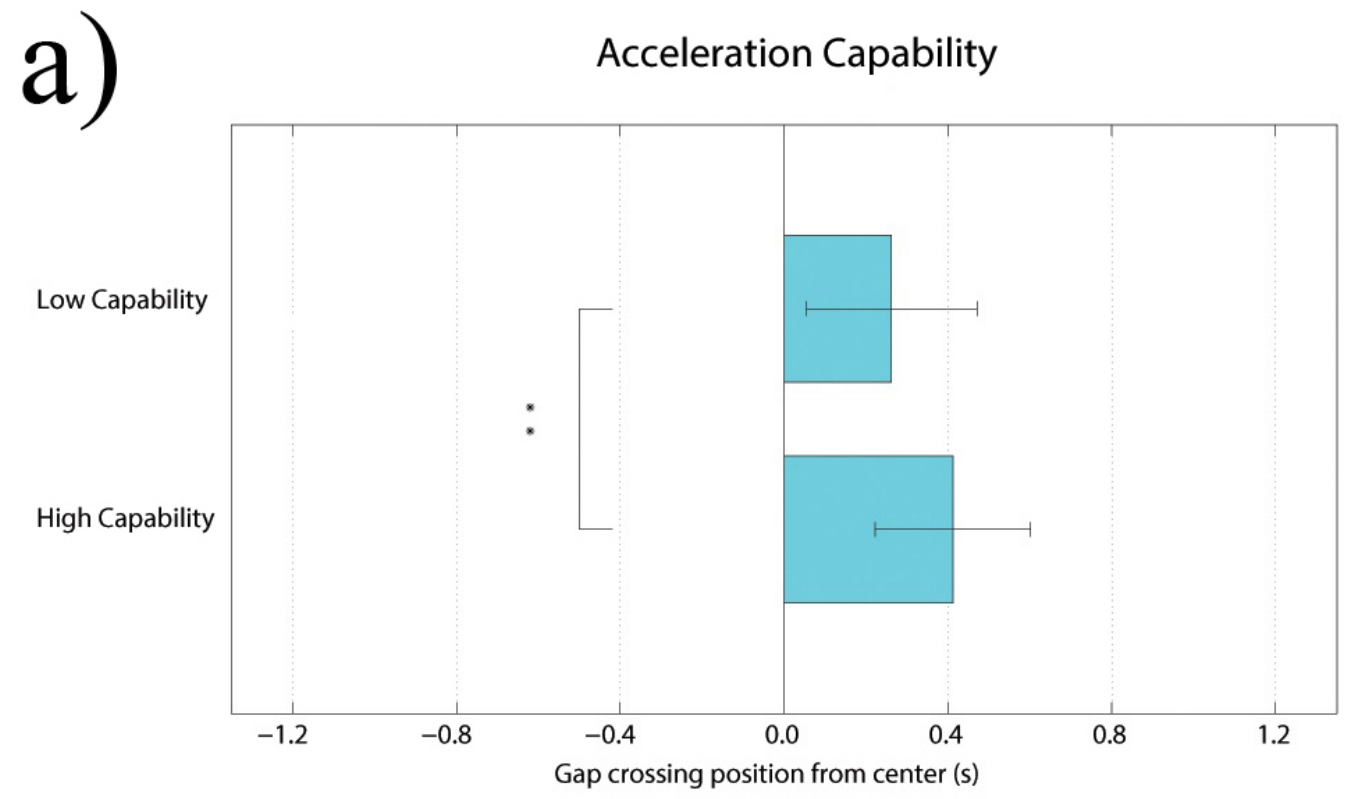

b)

Offset

Early Offset

No Offset

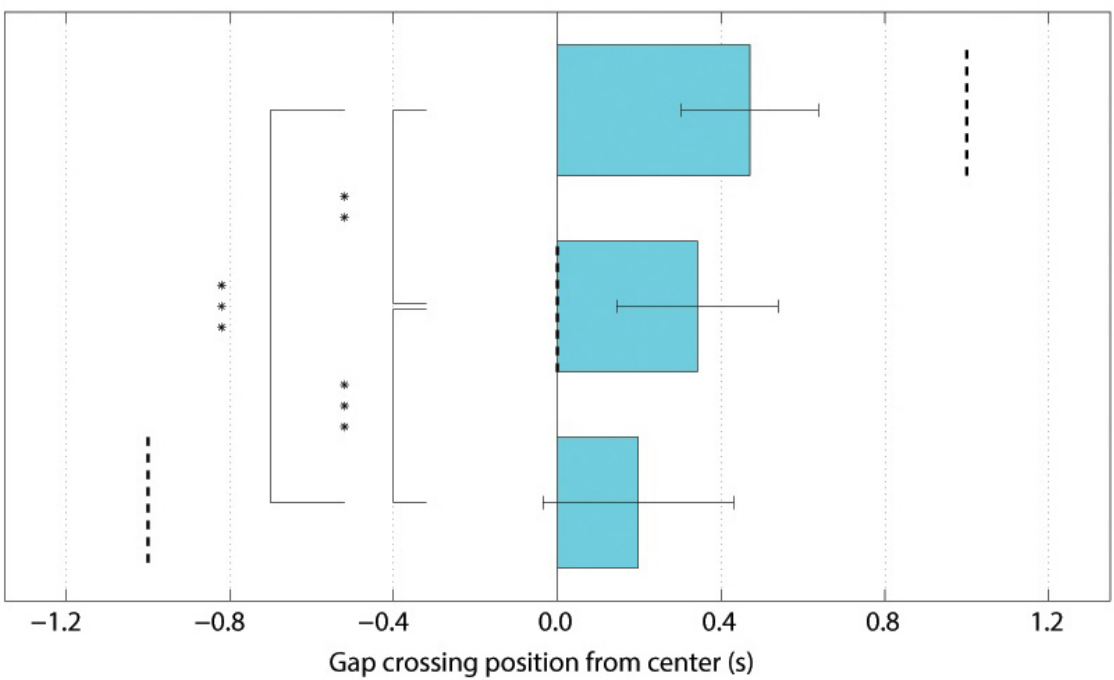

Time to Arrival

$6.5 \mathrm{~s}$

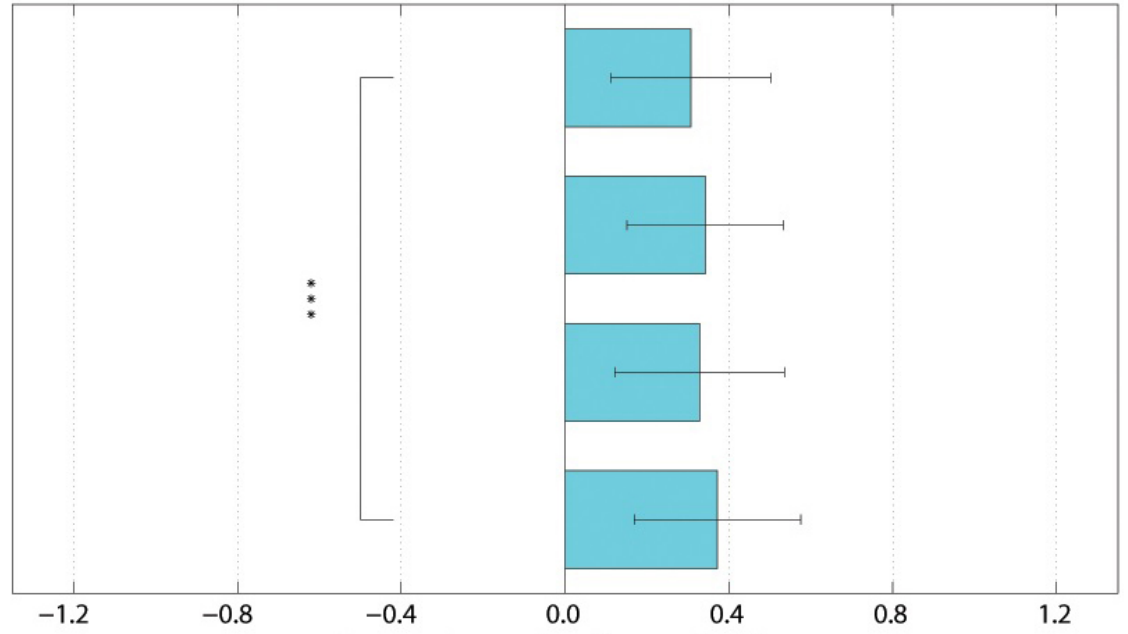

Gap crossing position from center (s) 
Figure 7

Current Speed
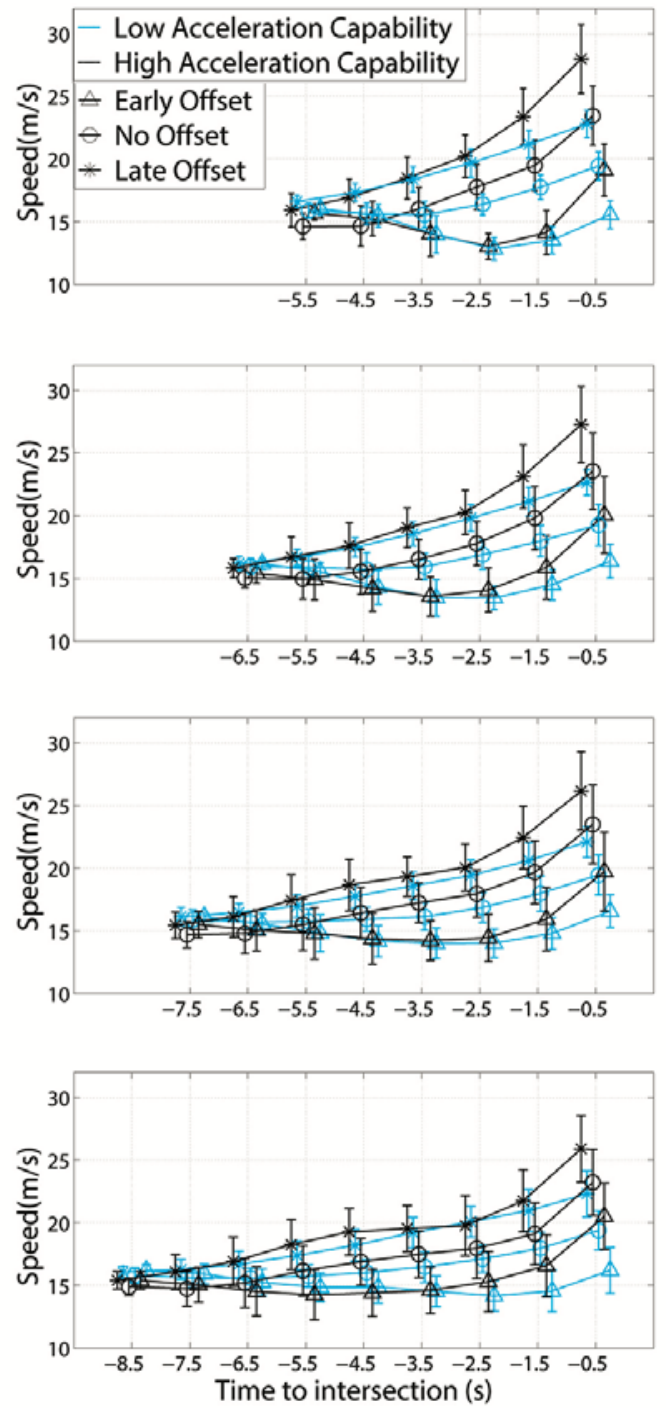

Current Deviation
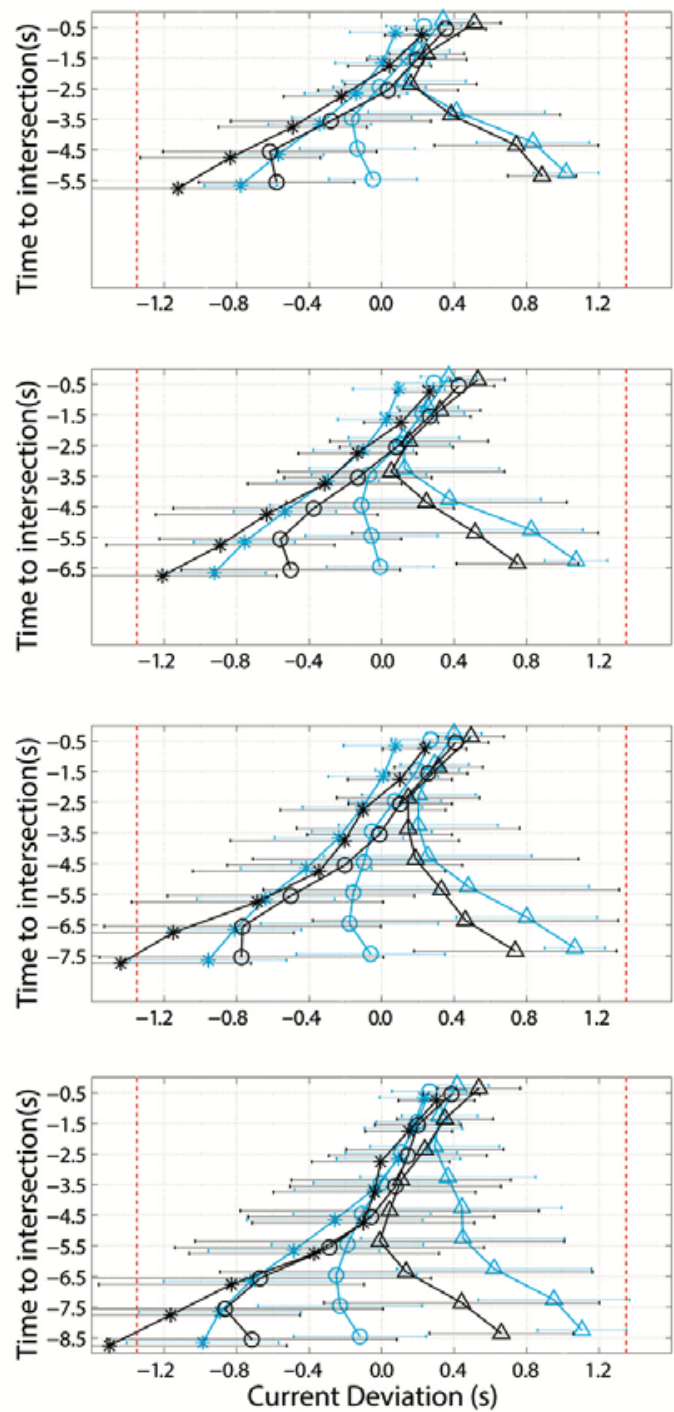
Figure 8

a)

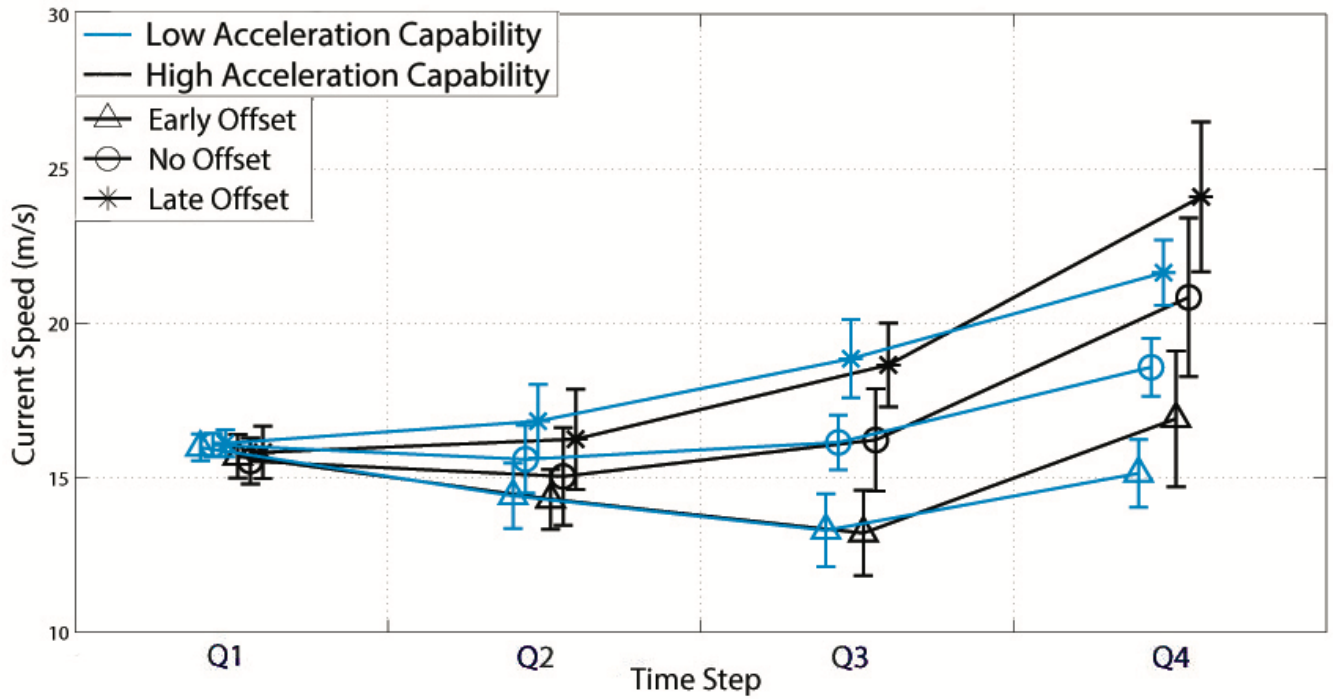

b)
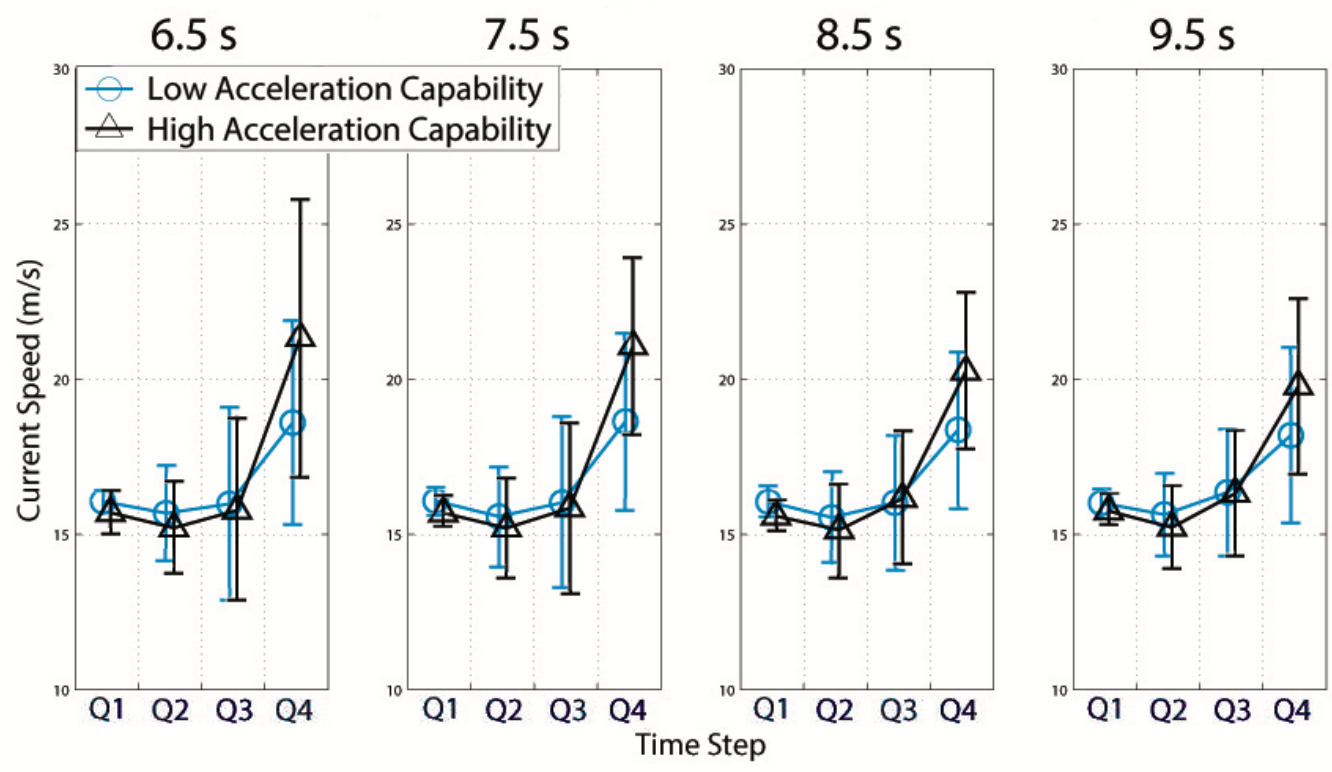

c)
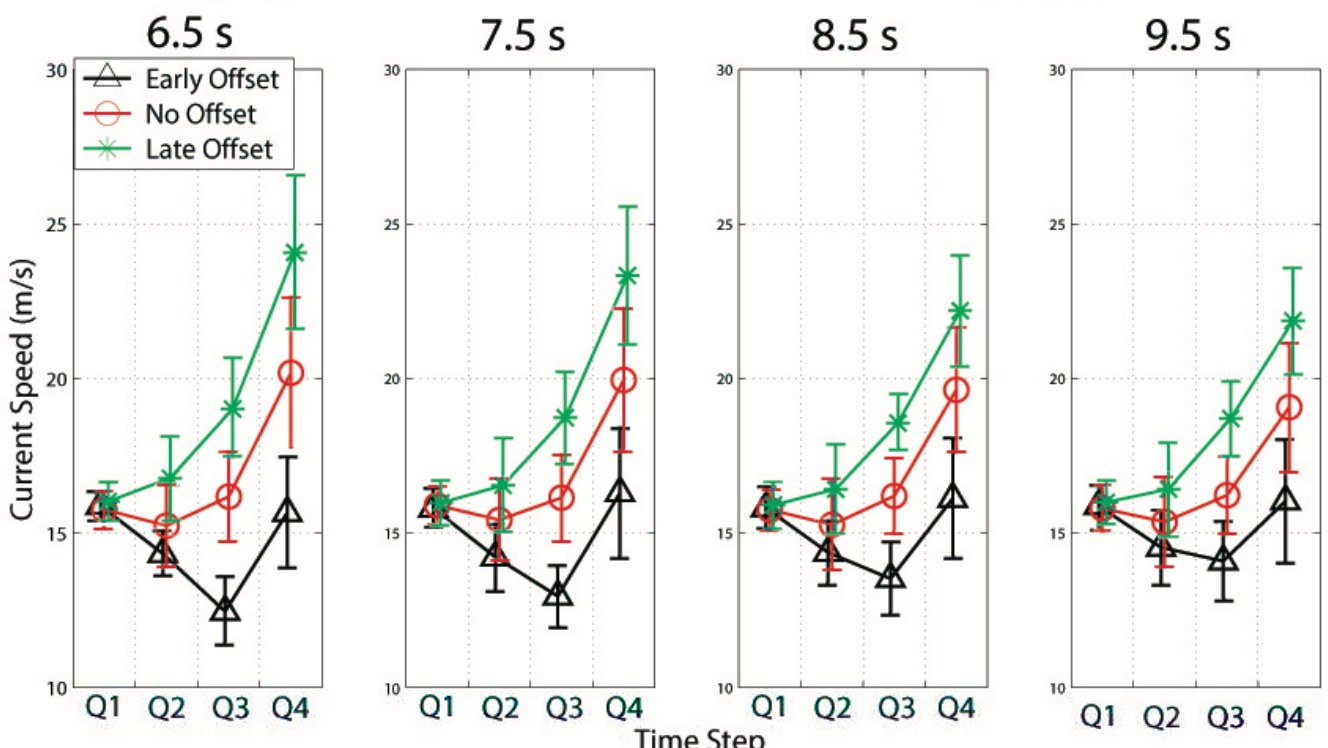
Figure 9

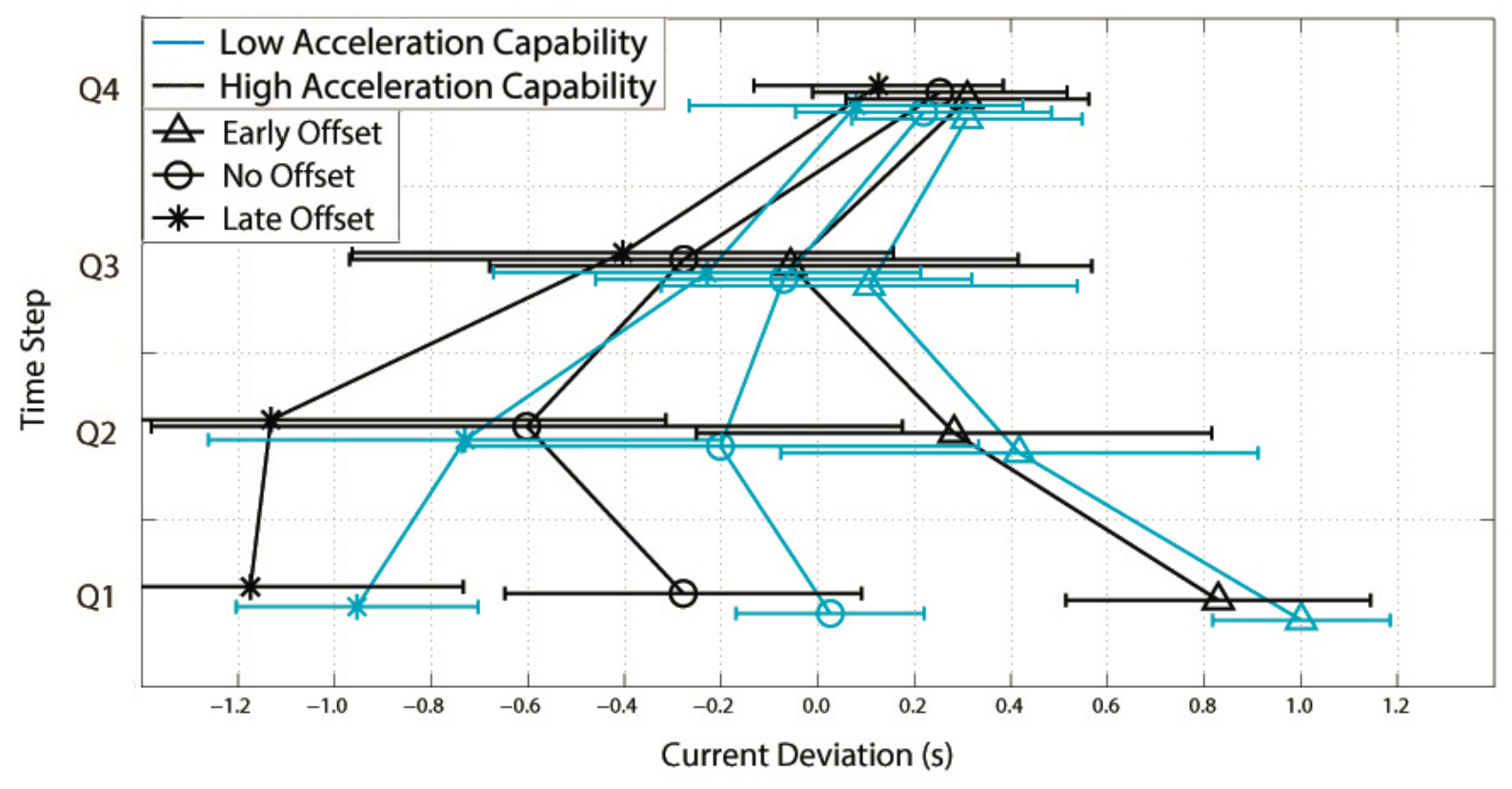


Figure 10

a)

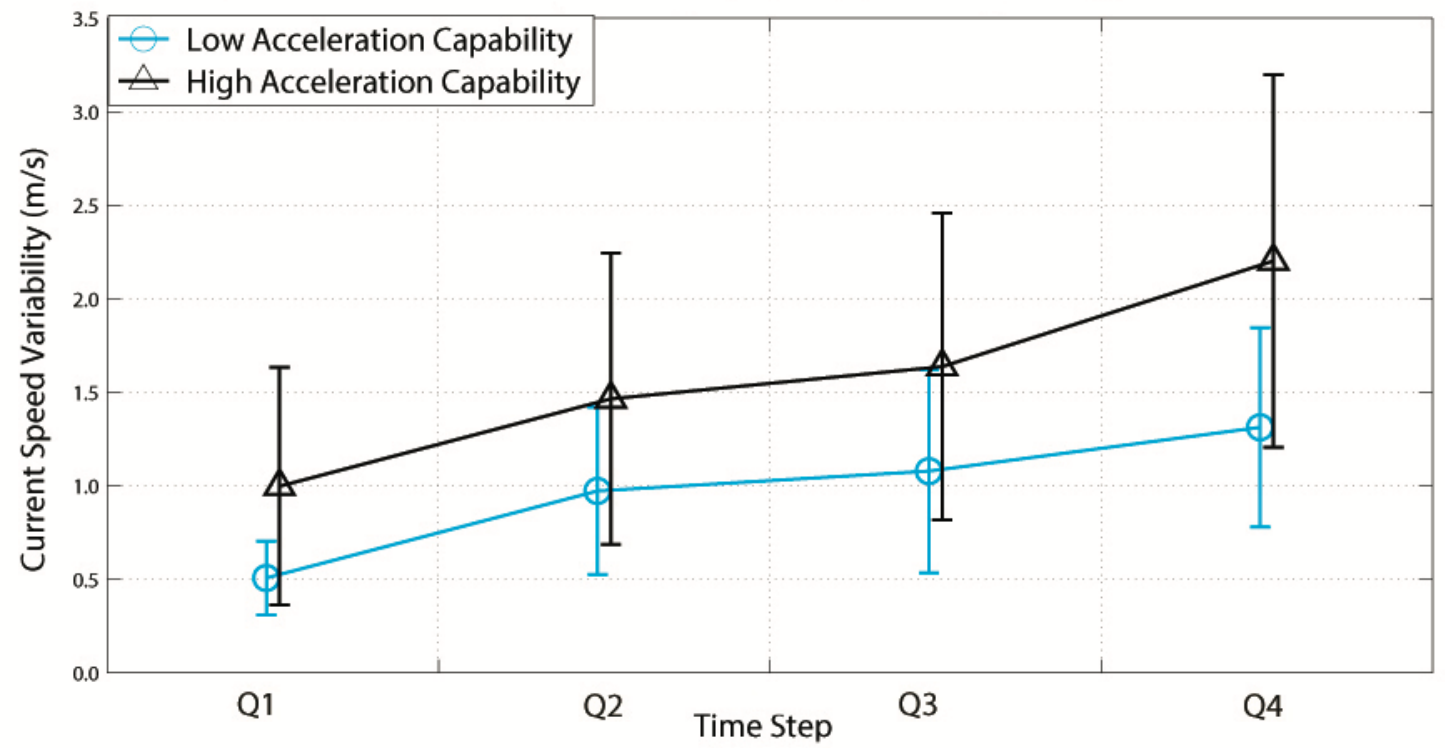

b)

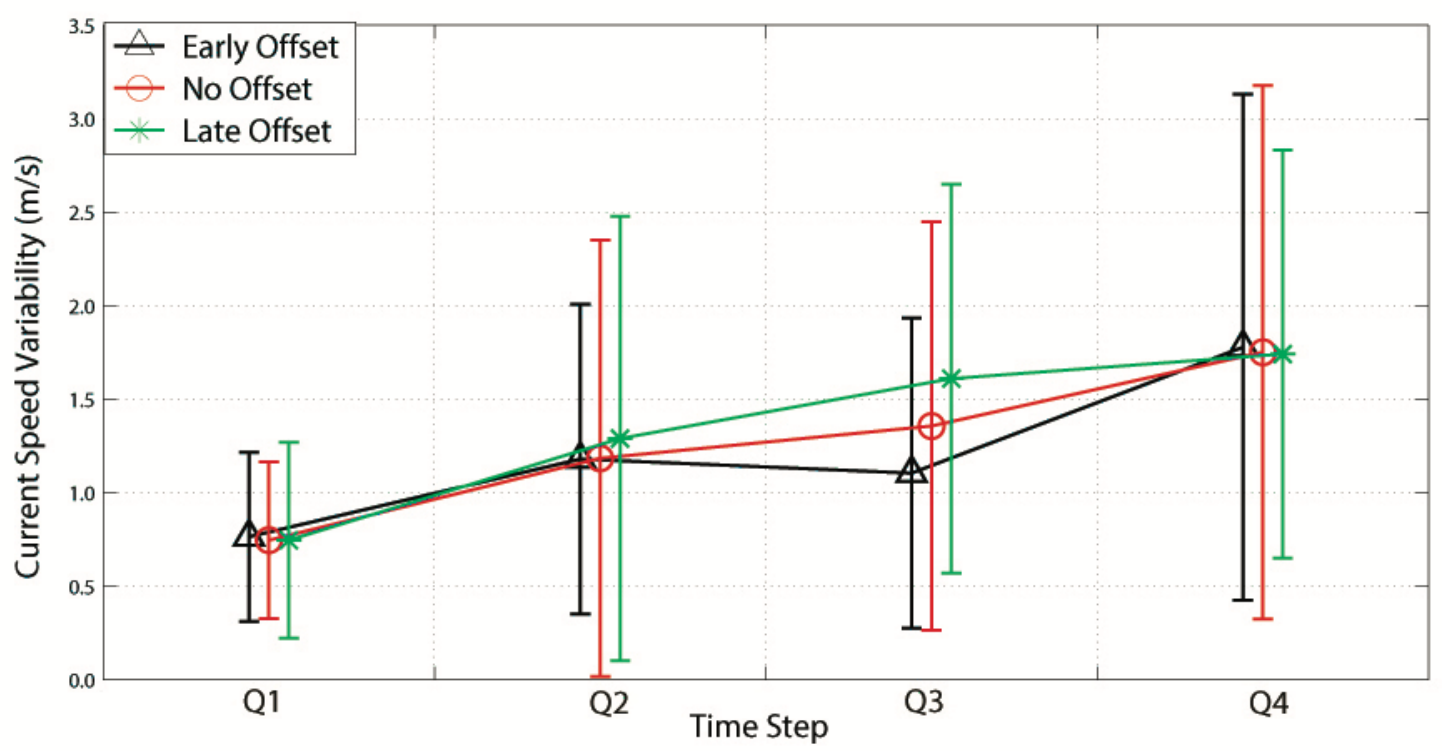


Figure 11

a)

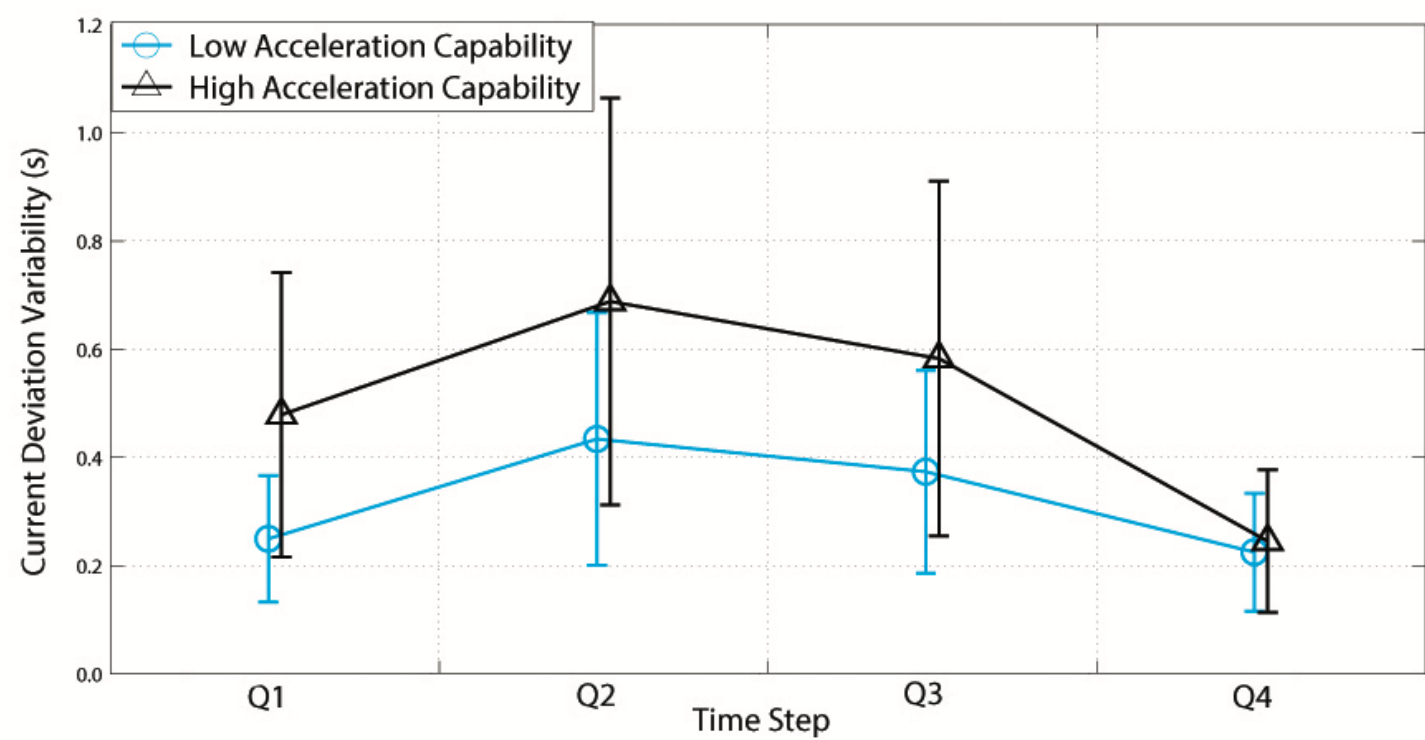

b)

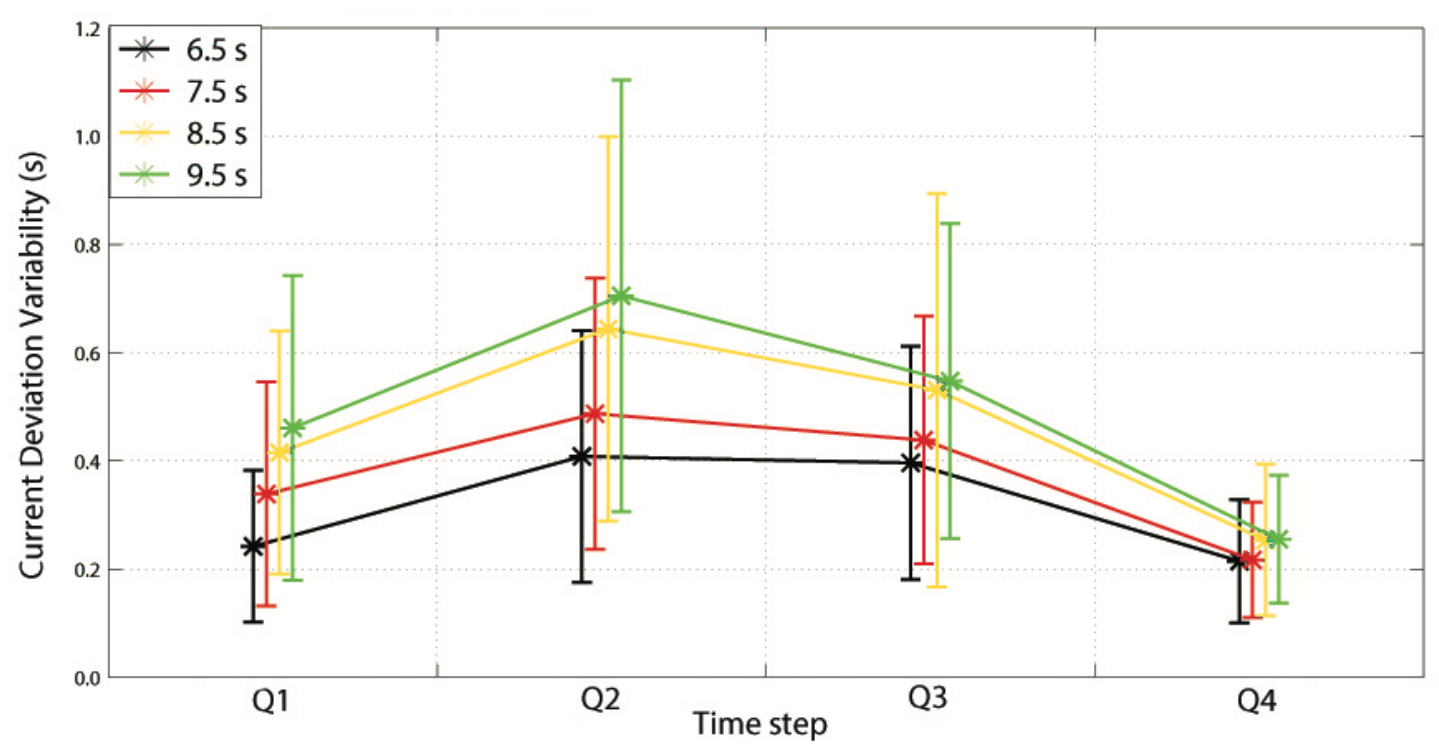

c)

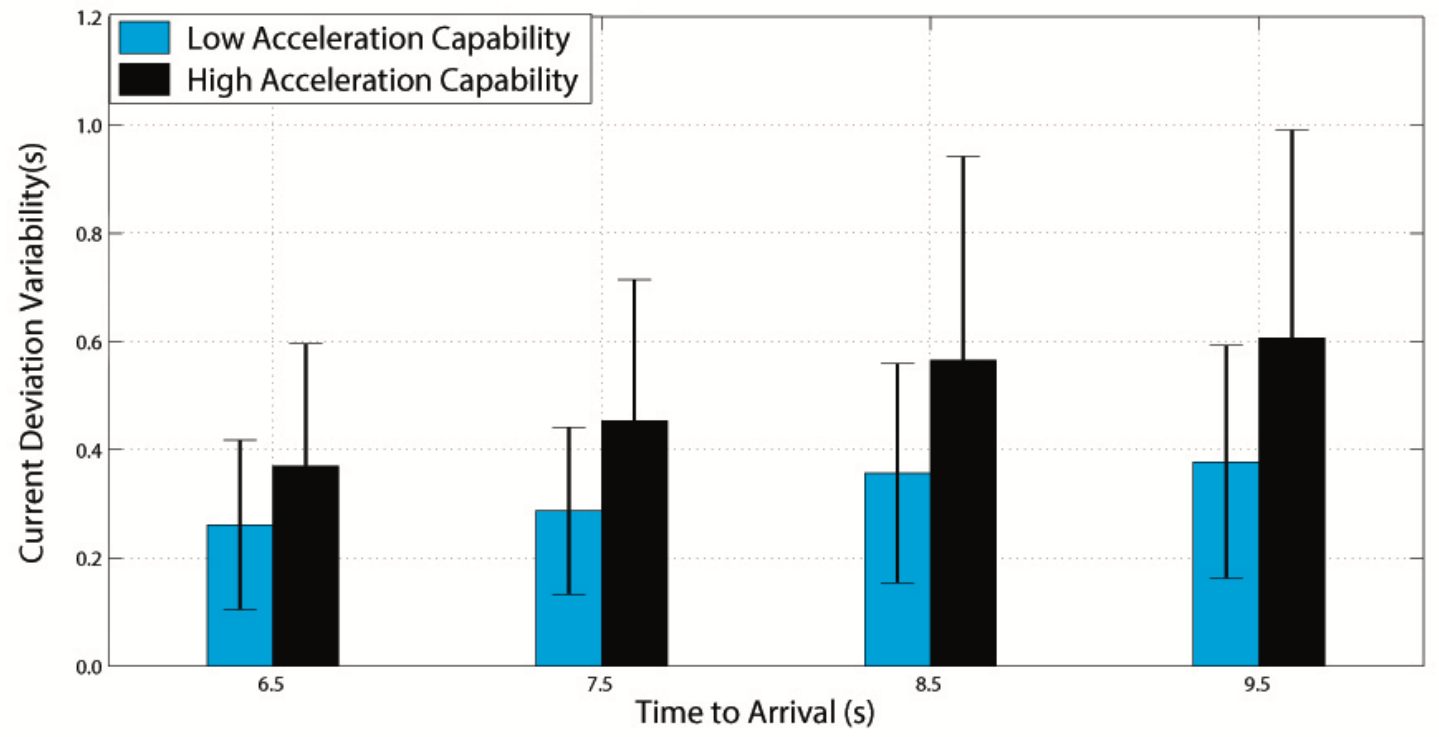


